\title{
Investigation of Theoretical Models for the Elastic Stiffness of Nanoparticle-Modified Polymer Composites
}

\author{
T. Thorvaldsen, ${ }^{1}$ B. B. Johnsen, ${ }^{1}$ T. Olsen, ${ }^{1}$ and F. K. Hansen ${ }^{1,2}$ \\ ${ }^{1}$ Norwegian Defence Research Establishment (FFI), P.O. Box 25, 2027 Kjeller, Norway \\ ${ }^{2}$ Department of Chemistry, University of Oslo, P.O. Box 1033 Blindern, 0315 Oslo, Norway \\ Correspondence should be addressed to T. Thorvaldsen; tom.thorvaldsen@ffi.no
}

Received 19 May 2015; Accepted 21 July 2015

Academic Editor: Abbas S. Milani

Copyright ( $) 2015$ T. Thorvaldsen et al. This is an open access article distributed under the Creative Commons Attribution License, which permits unrestricted use, distribution, and reproduction in any medium, provided the original work is properly cited.

\begin{abstract}
Mathematical models are investigated and suggested for the calculation of the elastic stiffness of polymer nanocomposites. Particular emphasis is placed on the effect on the elastic stiffness from agglomerates and the particle interphase properties. The multiphase Mori-Tanaka model and an interphase model are considered as two relevant models. These models only include and require the designation of a few system independent parameters with a clear physical meaning. Extensions of the models are also presented. The model calculations are compared to results from other models, as well as experimental data for different nanocomposites. For nanocomposites with spherical particles and with fiber-like particles, the suggested models are found to be the most flexible ones and are applicable to estimate the stiffness increase of nanocomposites for both low and high particle volume fractions. The suggested theoretical models can hence be considered as a general multiscale "model toolbox" for analysis of various nanocomposites.
\end{abstract}

\section{Introduction}

Polymers are widely used in engineering applications, such as in adhesives and in fiber-reinforced composites, where it constitutes the continuous phase. Model analysis of lightweight materials is becoming more important in the design, development, and analysis of novel and complex structures. There is often a desire or need to improve the mechanical properties of the polymers, especially for advanced high-performance applications. The traditional approach has been to add particulate inclusions of microsize, such as inorganic or rubber particles, to the polymers. In recent years, however, a lot of effort has gone into investigating the effect of adding nanosized inclusions [1-3]. A range of nanosized inclusions with different shape and chemical composition has been investigated, such as nanoclay, nanosilica, carbon nanotubes (CNTs), and carbon nanofibers (CNFs). Since the required loading level of the nanoparticles in the polymer is much lower than that for inclusions of microsize, many of the intrinsic properties of the polymer will be retained after the addition of nanoparticles.
One mechanical property that is of significant interest is the nanocomposite elastic stiffness, that is, Young's modulus of the nanocomposite. Most of the commonly used inclusions have a higher elastic modulus than the polymer itself and may therefore contribute to an increased elastic stiffness. The elastic stiffness of a nanocomposite material will, among others, depend on the type, orientation, and the volume fraction of the nanoparticles (assuming that the remaining constituents and the production process are kept constant).

The macroscopic nanocomposite material parameters are required in finite element (FE) modeling, among others. There may be a need for FE modeling of the nanocomposite itself or of a composite material where the nanomodified polymer constitutes the matrix of a continuous fiberreinforced material. It would therefore be of interest to establish a set of mathematical models that can be used to calculate the macroscopic elastic stiffness of various nanoparticlereinforced polymer composites.

For the models to be able to handle inclusions of various geometries, they must be flexible, while at the same time giving accurate predictions. In practice, the nanocomposite 
structure on the microlevel may be difficult to understand in detail. A detailed microstructural understanding may, however, not be required on a macroscopic level. Therefore, the mathematical models should have model parameters with physical meaning, without being directly system dependent, but they should still be sophisticated enough to give an accurate estimate and to predict the behavior observed in experimental testing.

The main aim of this work is to identify and establish flexible and system independent mathematical model tools for the prediction of the elastic stiffness of multiphase nanocomposites. The influence from the interphase around the nanoparticles and the influence from agglomerates are two effects that are of high interest to model for advanced and high-performance nanocomposites. Both effects will depend on the type of polymer, type of inclusion, loading level of the inclusion, and the processing conditions, and they thus have a practical interest for various nanoparticle/polymer systems. The main contribution from this work will therefore be more on defining a "model toolbox" rather than presenting new models for relevant nanocomposites. After the models are established and described, the model calculations are compared to experimental test data.

\section{Mathematical Models}

2.1. Nanoparticle/Polymer Characteristics. To actually increase, in this case, the elastic stiffness of a polymer material using particles of nanosize, several factors need to be considered and optimized. First of all, the nanoparticles are very small and therefore have a very large surface compared to larger particles; the surface area is inversely proportional to the particle size. The large surface area is, however, only available if the particles are well dispersed in the polymer. A good dispersion of the particles is believed to be a crucial requirement for obtaining a significant stiffness increase. If the particles form agglomerates, the inclusion phase will act more as defects/impurities in the homogenous polymer, which may instead reduce the bulk elastic stiffness $[4,5]$. For nanocomposites with well-dispersed particles, significant increase of the stiffness (and also other properties, such as the toughness) is often explained by interphase effects. A precise description of the mechanical properties and behavior of the interphase is very challenging. Zhang et al. [6] describe these effects as the formation of a "threedimensional physical network of interphase in a polymer matrix." For the interphase, there may be other chemical and physical properties than in the bulk matrix, with differences in, among others, the polymer morphology and chain conformation [7], as well as the adhesive properties between the particle and the matrix. Several factors have been shown to affect the binding properties, such as the functionalization or surface treatment of the particles [8] and/or the choice of curing agent [9]. The stiffness of the composite is, furthermore, depending on the geometry and the orientation of the particles. Particles, where the size in one direction is significantly different from the others, such as for CNTs and CNFs, will introduce directional dependent changes in stiffness even for particles considered as isotropic.
Also, composites with curved fiber-like particles (i.e., with waviness) have been reported to give a lower stiffness compared to composites with straight fiber-like particles [10].

With a relatively large set of properties, partly interrelated, that will affect and alter the elastic stiffness of a given nanoparticle/polymer system, a study purely based on experiments will soon become too complex, time consuming, and expensive. As mentioned above, the understanding and development of new nanocomposites will therefore benefit from having flexible and accurate mathematical models on macrolevel with only a few model parameters to vary. This set of models will then function as the multiscale mathematical "model toolbox" for understanding the behavior of the nanocomposites. It is, however, important to have a strong coupling to experimental test results for verification of the models.

2.2. Modeling Approaches. Fisher and Brinson [10] refer to two main mathematical multiscale modeling approaches for nanocomposites, that is, the "top-down" and the "bottomup" approach. In the "bottom-up" approach, one starts out with the atomistic structure of the nanoreinforcements and the matrix. Typical model techniques are quantum mechanics, molecular dynamics, and Monte-Carlo simulations [10, 11]. These simulations end up in fairly large systems to be solved for only a small part of the composite material, for example, a single CNT attached to a relatively short polymer chain. The output from these calculations should then be representative for the entire composite. Moreover, these simulations are very time consuming, even with large computing resources available.

The "top-down" approach, on the other hand, is based on continuum mechanics, where the polymer and the nanoand microinclusions are treated as continuum elements. The nanocomposite models often use short-fiber models and laminate theory [12] as a starting point. Equivalent models are then established for particles of smaller size. Additional effects, such as interphase effects, are often incorporated in the equivalent models, since these effects seem to be more significant when reducing the size of the particle. One main benefit of the "top-down" approach is that one is able to calculate for a larger part of the composite material (often referred to as a representative volume entity) to estimate the macroscopic properties of the materials.

Due to the size of the nanoparticle filler material, care must be taken when applying the continuum mechanics approach since the structure and interactions at the atomistic level are essential for a precise description of the mechanical properties. For this study, the "top-down" approach, employing continuum mechanics, is, however, assumed to be a valid approach.

2.3. Analytical Models. Several analytical models for the elastic stiffness of nanocomposites are based on the pioneer work by Cox [13]. Rule of mixtures models specially designed for nanocomposites with nonspherical particle geometry have also been presented [14]. Moreover, to include a random orientation of the particles, models for weighting of the properties for the different orientations have been defined, 
for example [12]. Approaches employing laminate theory in describing the macroscopic properties of the composite have also been considered; see, for example, [15] and the references therein.

Most of these more "traditional" short-fiber based models assume a perfect dispersion of the particles in the matrix, that is, one inclusion phase in addition to the matrix phase, and a no-slip boundary condition at the particle-matrix interface. Moreover, the traditional models are often developed for a specific nanocomposite, that is, one type of nanoparticle with a given geometry, embedded in a given polymer system. The model parameters are then specific to the particular nanoparticle/polymer system and may not be transmissible to other nanocomposites. More flexible models with less system dependent parameters are therefore appropriate to establish.

In the following, a set of more flexible models for nanocomposites are described: the Mori-Tanaka approach and an interphase model. For comparison, the Halpin-Tsai equation and a slip/no-slip model are also included since these latter models are often applied in comparison with experimental data. It should also be mentioned at this point that expressions based on the Mori-Tanaka method have been established for specific nanocomposites and applied for comparison with experimental results [16-22], but, again, these expressions are not as flexible as the more general multiphase model.

2.3.1. The Halpin-Tsai Equation. For aligned particles, the Halpin-Tsai equations, which are based on empirical data, are often applied [23-26]. For the special case of spherical particles, these equations are reported in the literature to give a reliable estimate for the stiffness properties of the nanocomposite and are often applied for comparison with experimental test results. The modified version of the HalpinTsai equations has also been presented, including an orientation factor $\beta$ that takes into account the randomness of discontinuous fibers.

The modified version of the Halpin-Tsai equation for the longitudinal elastic modulus can be expressed as [27, 28]

$$
\begin{aligned}
E_{C} & =\frac{1+\alpha \eta V_{f}}{1+\eta V_{f}} E_{m}, \\
\eta & =\frac{\left(\beta E_{f} / E_{m}\right)-1}{\left(\beta E_{f} / E_{m}\right)+2 \alpha}
\end{aligned}
$$

where in the above expression $E_{C}$ is the elastic stiffness of the composite, $E_{f}$ and $E_{m}$ are the elastic stiffness of the inclusions and the matrix, respectively, and $\alpha=l / d$ is the aspect ratio, $l$ being the length and $d$ being the diameter of the particle. In the original Halpin-Tsai equation, $\beta$ equals unity. For composites with a three-dimensional random orientation of fiber-like particles (where $l \neq d$ ), $\beta=1 / 6$ is used.

2.3.2. The Mori-Tanaka Method. To include more than one inclusion phase, for example, the combination of dispersed nanoparticles and voids/agglomerates, or a second type of particle with other elastic properties and/or a different geometric shape, more general multiphase models are established. The Mori-Tanaka method is one such attempt [29-31], which also has been reported to agree well with experimental results; see, for example, [10] and the references therein. Different model variants based on the Mori-Tanaka approach can be found in the literature; see, for example, [32] for a review.

The composite elastic stiffness $C_{C}$ in the Mori-Tanaka model for a multiphase composite with unidirectionally aligned inclusions can generally, following the derivation in [10], be expressed as

$$
\begin{aligned}
C_{C} & =V_{0} C_{0} A_{0}+\sum_{r=1}^{N-1} V_{r} C_{r} A_{r} \\
& =\left(V_{0} C_{0}+\sum_{r=1}^{N-1} V_{r} C_{r} A_{r}^{\mathrm{dil}}\right)\left(V_{0} I+\sum_{r=1}^{N-1} V_{r} A_{r}^{\mathrm{dil}}\right)^{-1} \\
& =\left(V_{0} C_{0}+\sum_{r=1}^{N-1} V_{r} C_{r} A_{r}^{\mathrm{dil}}\right) A_{0}
\end{aligned}
$$

where

$$
\begin{aligned}
A_{r}^{\text {dil }} & =\left[I+S_{r} C_{r}^{-1}\left(C_{0}-C_{r}\right)\right]^{-1}, \\
A_{0} & =\left[V_{0} I+\sum_{r=1}^{N-1} V_{r} A_{r}^{\mathrm{dil}}\right]^{-1}, \\
A_{r} & =A_{r}^{\mathrm{dil}} A_{0} .
\end{aligned}
$$

In the above expressions, phase 0 is the continuous and homogeneous matrix phase, and phases 1 to $N-1$ are the inclusion phases. Moreover, $V_{0}$ is the volume fraction of the matrix phase, $C_{0}$ contains the stiffness properties of the matrix phase, $V_{r}$ is the volume fraction of the $r$ th inclusion phase, and $C_{r}$ contains the stiffness properties of the $r$ th inclusion phase. The quantity $I$ is the identity matrix and $S_{r}$ is the (second-order) Eshelby tensor for the $r$ th inclusion phase $[30,31]$. For composites where the particles can be modeled as having a spheroidal shape (e.g., spherical, oblate, or prolate shape) and included in a homogeneous infinite matrix, the Eshelby tensor is constant. Expressions for the Eshelby tensor for relevant spheroidal inclusion geometries can be found in the literature, for example, $[18,22,33]$.

For the special case of a two-phase composite, including the matrix (phase 0) and the nanoparticles (phase 1), the stiffness relation in (2) can be expressed as

$$
C_{C}=\left(V_{m} C_{m}+V_{p} C_{p} A_{p}^{\mathrm{dil}}\right)\left(V_{m} I+V_{p} A_{p}^{\mathrm{dil}}\right)^{-1},
$$

where now $m$ indicates the matrix phase and $p$ indicates the inclusion phase. Furthermore, $V_{m}$ is the volume fraction of the matrix, $V_{p}$ is the volume fraction of the inclusions, $C_{m}$ is the stiffness matrix of the matrix, $C_{p}$ is the stiffness matrix of the inclusions, $I$ is the identity matrix, and

$$
A_{p}^{\text {dil }}=\left[I+S_{p} C_{p}^{-1}\left(C_{m}-C_{p}\right)\right]^{-1},
$$


where $S_{p}$ is the (second-order) Eshelby tensor for the inclusion phase.

The Mori-Tanaka model for a multiphase composite with randomly oriented inclusions can in a similar way be expressed as

$$
\begin{aligned}
& C_{C, \text { random }} \\
& =\left(V_{0} C_{0}+\sum_{r=1}^{N-1} V_{r}\left\{C_{r} A_{r}^{\mathrm{dil}}\right\}\right)\left(V_{0} I+\sum_{r=1}^{N-1} V_{r} A_{r}^{\mathrm{dil}}\right)^{-1} \\
& =\left(V_{0} C_{0}+\sum_{r=1}^{N-1} V_{r}\left\{C_{r} A_{r}^{\mathrm{dil}}\right\}\right) A_{0},
\end{aligned}
$$

where the curly brackets indicate orientational averaging of all possible orientations, as described and shown in [10] (and also in [34] for another orientation of the local axes of the inclusion compared to that used in [10]). In the same way as for aligned inclusions, the elastic stiffness may for a two-phase composite be written as

$$
C_{C, \text { random }}=\left(V_{m} C_{m}+V_{p}\left\{C_{p} A_{p}^{\mathrm{dil}}\right\}\right)\left(V_{m} I+V_{p} A_{p}^{\mathrm{dil}}\right)^{-1} .
$$

For the special case of a three-phase composite, the contribution from the second inclusion phase is added to the expressions for aligned and random oriented composites, respectively.

An extension of the above model, also taking into account the curvature (waviness) of the fiber-like particles, has been included by performing a finite element method model calculation for a single curved fiber-like particle surrounded by matrix $[10,35]$. Curved fiber-like particles have a reduced stiffness contribution to the composite compared to straight fiber-like particles.

2.3.3. Interphase Models. As for the rule of mixtures and short-fiber composite expressions described above, the MoriTanaka method assumes a no-slip condition at the interface between the particles and the surrounding matrix. Such an interface interaction condition may not be a correct description for all nanoparticle/polymer systems.

One approach for allowing a slippage at the interface is presented by Lewis and Nielsen [36] and McGee and McGullough [37]. In their model, the generalized Einstein coefficient $\left(k_{E}\right)$ is altered for the "no-slip" and "slippage" conditions. This model parameter, however, has to be estimated for each nanoparticle/polymer system, which makes the model less flexible for estimating the elastic stiffness of different systems.

As an alternative approach, it can be assumed that the binding characteristics can be expressed in the form of an elastic stiffness for the interphase surrounding the particles, that is, a "second matrix phase." The stiffness will typically vary (radially) through the interphase, with a smooth transition from the particle surface to the surrounding bulk polymer. As pointed out by Fornes and Paul [7], when employing a continuum mechanics approach, one should assume that each constituent material in the composite does not affect or alter the properties of the other constituents. The interphase should thus be defined as a geometrically

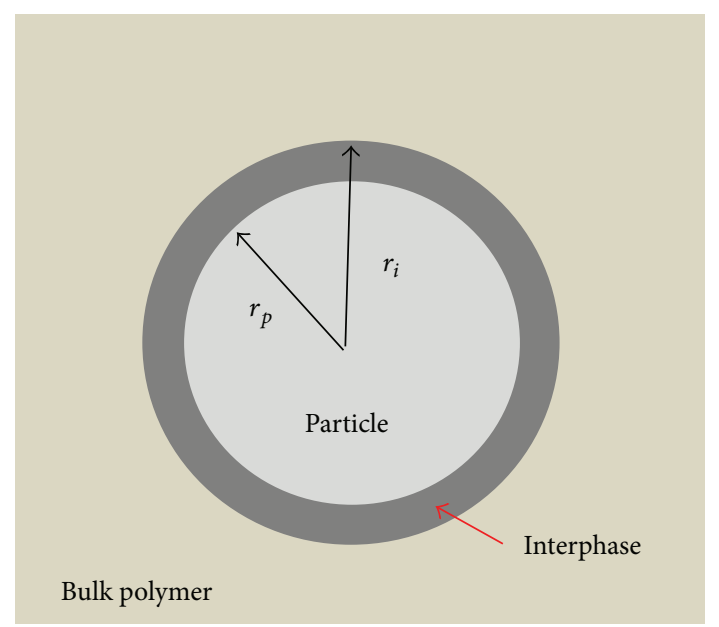

FIGURE 1: Representative volume entity (RVE) for the particle with interphase. The radius of the particles is $r_{p}$, whereas the radius of the interphase is $r_{i}, r_{i} \geq r_{p}$.

well-defined region with given elastic properties. A high interphase elastic stiffness indicates good bonding and less flexibility to deform when loaded, whereas a lower inclusion phase elastic stiffness indicates a weaker bonding with more flexibility to deform when loaded.

Measurements of the interphase elastic properties have been done, for example [38]. Since it may be difficult to perform stiffness measurements of the interphase without including the stiffness of the particle itself, molecular model calculations have instead been applied for estimating the interphase properties, for example [39]. Furthermore, the interphase properties may vary as a function of the volume fraction of nanoparticles in the composite. This change in interphase properties may thus indicate a variation in the thickness of the interphase surrounding the particles. Alternatively, the variation of the properties in the interphase can be due to variation in elastic stiffness. Tuning the elastic stiffness and the interphase thickness can therefore give an adequate description of the interphase properties and behavior. The interphase properties are discussed further in Section 4.1.

An effective interphase model has been presented by Odegard et al. [39, 40]. In this case, a no-slip condition at the interface between the particle and the interphase and between the interphase and the bulk matrix has been assumed. However, the interphase itself introduces the wanted flexibility. Note that Odegard et al. refer to their model as an "effective interface model." To the authors' knowledge and understanding, the interface is the surface of the particle being in contact with its surroundings. The interphase, on the other hand, is the polymer matrix region surrounding the particle. A sketch is given in Figure 1 for a spherical particle with radius $r_{p}$, surrounded by an interphase with thickness $t_{i}=r_{i}-r_{p}, r_{i} \geq r_{p}$. This latter terminology is also in accordance with Fisher and Brinson [10] and will be employed in this paper. 
In the interphase model by Odegard et al., the elastic stiffness of the nanoparticle/polymer composite $C_{C}$, where the particles have a surrounding interphase and a bulk matrix phase outside the interphase, can be expressed as

$$
\begin{aligned}
C_{C} & =C_{m} \\
+ & {\left[\left(V_{p}+V_{i}\right)\left(C_{i}-C_{m}\right) A_{p i}+V_{p}\left(C_{p}-C_{i}\right) A_{p}\right] } \\
& \cdot\left[V_{m} I+\left(V_{p}+V_{i}\right) A_{p i}\right]^{-1},
\end{aligned}
$$

where in this case

$$
\begin{aligned}
A_{p} & =I-S_{p}\left[S_{p}+\left(C_{p}-C_{m}\right)^{-1} C_{m}\right]^{-1} \\
A_{p i} & =I-S_{p}\left\{\frac{V_{p}}{V_{i}+V_{p}}\left[S_{p}+\left(C_{p}-C_{m}\right)^{-1} C_{m}\right]^{-1}\right. \\
& \left.+\frac{V_{i}}{V_{i}+V_{p}}\left[S_{p}+\left(C_{i}-C_{m}\right)^{-1} C_{m}\right]^{-1}\right\}
\end{aligned}
$$

In the same way as for the Mori-Tanaka model described in the previous section, $m$ indicates the matrix phase and $p$ indicates the inclusion phase, whereas $i$ indicates the interphase. Furthermore, $V_{m}$ is the volume fraction of the matrix, $V_{p}$ is the volume fraction of the inclusions, and $V_{i}$ is the volume fraction of the interphase. Moreover, $C_{m}$ is the stiffness matrix of the matrix, $C_{p}$ is the stiffness matrix of the inclusions, $C_{i}$ is the stiffness matrix of the interphase, and $I$ is the identity matrix. It should at this point also be noted that the geometric properties of the interphase are not explicitly given in the stiffness expressions in (8) and (9), but implicitly through the volume fraction calculations of the different constituents.

In the model presented by Odegard et al., the particles are assumed to be spherical (and thus aligned), and $S_{p}$ hence refers to the Eshelby tensor for spheres. An extension of the Odegard et al. model is to include spheroidal shapes different from spheres (i.e., prolate and oblate spheroidal) and also random orientation of the inclusions. Including other spheroidal shapes is easily done by replacing the Eshelby tensor for spheres with the tensor for another spheroidal shaped inclusion. The random distribution may be more complex. In this paper, it is assumed that the same "averaging strategy" as was performed for the multiphase Mori-Tanaka model is applicable. By comparing the terms in the MoriTanaka model and the effective interphase model, we find the terms that are required to be orientationally averaged. The stiffness for such a composite can then be expressed as

$$
\begin{aligned}
C_{C} & =C_{m}+\left[\left(V_{p}+V_{i}\right)\left\{\left(C_{i}-C_{m}\right) A_{p i}\right\}\right. \\
& \left.+V_{p}\left\{\left(C_{p}-C_{i}\right) A_{p}\right\}\right] \cdot\left[V_{m} I+\left(V_{p}+V_{i}\right) A_{p i}\right]^{-1},
\end{aligned}
$$

where the curly brackets indicate the average of the quantity over all possible orientations. To the authors' knowledge, the model extensions have not been published elsewhere.
2.3.4. Agglomerate Models. Agglomeration is highly relevant for nanocomposites with nonspherical particles since the particles in such cases are more likely to be entangled prior to the dispersion process. This situation can be handled by the multi-phase Mori-Tanaka model described in Section 2.3.2, by assuming that one of the particulate phases is an agglomerate phase.

Special models are also established for certain composites including a combination of free and agglomerated particles. As a first example, a model for composites with carbon nanotubes (CNTs) in a polymer system has been presented by Shi et al. [41, 42]. In their work, models are presented both for perfectly dispersed CNTs and for composites with a combination of dispersed particles and agglomerates of particles. In both approaches, the Mori-Tanaka method is employed. In their agglomerate model, the areas with concentrated CNTs are considered to be spherical inclusions with different elastic properties compared to the surrounding material. Two parameters are employed for describing the agglomeration. Based on the Voigt model, found in [43], and assuming that both the matrix and the CNTs have isotropic material properties, expressions for the elastic properties of the inclusions and the material outside the inclusions are established.

As a second example, Gershon et al. [5] presented an agglomerate model for CNFs in a polymer system. In their model, expressions are given for the volume fraction of agglomerated CNFs

$$
V_{\text {CNFagglomerate }}=\frac{E_{\text {agglomerate }} V_{\text {agglomerate }}}{E_{\mathrm{CNF}}},
$$

for the volume fraction of dispersed CNFs in the polymer, referred to as the matrix,

$$
V_{\mathrm{CNFmatrix}}=\frac{E_{\text {matrix }}-E_{\text {polymer }}}{E_{\mathrm{CNF}}-E_{\text {polymer }}},
$$

and finally for the composite

$$
\begin{aligned}
& E_{\text {composite }} \\
& =\frac{1}{\left(1-V_{\text {agglomerate }}\right) / E_{\text {matrix }}+V_{\text {agglomerate }} / E_{\text {agglomerate }}} .
\end{aligned}
$$

Experimental data for Young's modulus of the matrix, that is, the polymer with free and dispersed CNFs, and Young's modulus for the agglomerates are required. The values vary as a function of the volume fraction of CNFs.

\section{Experimental Work}

Experimental work was conducted with the main aim of comparing the experimental results with the model calculations (see Section 5) and to obtain realistic model parameters. In this work, the elastic stiffness variation of two silica/epoxy nanocomposite materials, upon increasing nanosilica content, was determined. 
3.1. Materials. Two different epoxy polymers were investigated in this work: one amine-cured and one anhydridecured system [44]. The amine-cured polymer system was Araldite LY 556/XB 3473, while the anhydride-cured polymer system was Araldite LY 556/Aradur 917/Accelerator DY 070. Both systems are from Huntsman. LY 556 is a standard bisphenol A based epoxy resin with an epoxy equivalent weight (EEW) of 183-189 g/eq. XB 3473 is an amine hardener containing two different diamines. The active hydrogen equivalent weight (AHEW) of XB 3473 is $\approx 43$ g/eq. Aradur 917 is a methyltetrahydrophthalic acid anhydride hardener with an AHEW of $166 \mathrm{~g} / \mathrm{eq}$, whereas DY 070 is an imidazole accelerator.

A sol of silica $\left(\mathrm{SiO}_{2}\right)$ nanoparticles in an epoxy resin was employed to produce the silica/epoxy nanocomposites. The Nanopox F400 was supplied by Evonik Hanse, Geesthacht, Germany. The silica phase in Nanopox F400 consists of surface-modified silica spheres with an average particle diameter of $20 \mathrm{~nm}$ and a narrow particle size distribution. The silica content is $40 \mathrm{wt} \%$, and the density of the silica $\rho_{r}$ is $2100 \mathrm{~kg} / \mathrm{m}^{3}$ [45]. The silica particles have an elastic stiffness of $70 \mathrm{GPa}$ and a Poisson ratio of 0.20 . The epoxy phase is a bisphenol A diglycidyl ether, and the EEW for Nanopox F400 is $299 \mathrm{~g} / \mathrm{eq}$. Nanopox F400 has a comparatively low viscosity, due to the low degree of agglomeration of the nanoparticles in the resin.

3.2. Specimen Preparation. Plates of the neat epoxy polymers were prepared by first mixing stoichiometric ratios of epoxy resin and hardener (and the accelerator in the case of the anhydride-cured system). After mixing, the blends were stirred manually using a spatula, thereafter heated to $80^{\circ} \mathrm{C}$ to lower the viscosity, and then stirred thoroughly again. Finally, the blends were vacuum degassed and then cast in a preheated metal mold that had been coated with a release agent. The resulting plate thickness was $4 \mathrm{~mm}$. The curing cycles employed were (1) 2 hours at $120^{\circ} \mathrm{C}, 2$ hours at $140^{\circ} \mathrm{C}$, and 2 hours at $180^{\circ} \mathrm{C}$ for the amine-cured system and (2) 4 hours at $80^{\circ} \mathrm{C}$ and 8 hours at $140^{\circ} \mathrm{C}$ for the anhydride-cured system.

The procedure for producing plates of the silica/epoxy nanocomposites was similar to that of the neat epoxy polymers, except that Nanopox F400 was also mixed into the blends. Plates with four different loadings of silica for each polymer system were prepared. The cured composite plates were visually observed to be free of air and blisters.

The density of the neat epoxy polymers $\rho_{m}$ and the nanocomposites $\rho_{c}$ was measured according to the immersion method described in ASTM D 792-08 [46]. Measurements were conducted on six pieces from each produced plate. The density was in the range from 1160 to $1315 \mathrm{~kg} / \mathrm{m}^{3}$ for the amine-cured system and in the range from 1200 to $1305 \mathrm{~kg} / \mathrm{m}^{3}$ for the anhydride-cured system. Based on the known weight fractions of silica in the nanocomposites, the density measurements were used to calculate the volume fraction $V_{p}$.

3.3. Material Characterization. Tensile testing was conducted on dumbbell specimens that were machined from the neat epoxy and silica/epoxy nanocomposite plates. The testing was conducted according to the relevant ISO standard [47, 48] on a Zwick BZ2.5/TN1S material testing machine, employing specimens of type 1BA. The test speed was $1 \mathrm{~mm} / \mathrm{min}$, and the strain was recorded using a clip-gauge extensometer. Values for the tensile modulus of elasticity $E_{t}$, the maximum tensile stress, or the tensile strength $\sigma_{m}$, and the maximum elongation, or tensile strain at break $\varepsilon_{b}$, were determined. The tensile modulus $E_{t}$ was determined from the linear part of the stress-strain curve in the strain range from $0.05 \%$ to $0.25 \%$. Average values of six replicate specimens are reported. The ambient temperature during the testing was $23 \pm 1^{\circ} \mathrm{C}$.

Dynamic mechanical analysis (DMA) was performed on a DMA 2980 Dynamic Mechanical Analyzer from TA Instruments. Rectangular specimens with the dimensions $3 \mathrm{~mm} \times 10 \mathrm{~mm} \times 60 \mathrm{~mm}$ were cut from the produced plates. The analysis was conducted in a three-point bending mode employing a low friction three-point bending clamp with a specimen free length of $50 \mathrm{~mm}$. The oscillation frequency was $1 \mathrm{~Hz}$, the preload force was set to $0.05 \mathrm{~N}$, and the "Force Track" was set to $150 \%$. The heating rate was $3^{\circ} \mathrm{C} / \mathrm{min}$. The value of the storage modulus, $E^{\prime}$, was measured at a temperature of $30^{\circ} \mathrm{C}$, and the value of the glass transition temperature $T_{g}$ of the epoxy polymer is reported as the peak value of the loss modulus, $E^{\prime \prime}$. Average values of three replicate specimens are reported.

\subsection{Experimental Test Results}

3.4.1. Amine-Cured System. The results from the tensile testing and the DMA of the neat amine-cured epoxy and its silica/epoxy nanocomposites are shown in Table 1 . The tensile testing showed a linear increase in the elastic modulus $E_{t}$ with increasing content of silica. The value increased from $2.61 \mathrm{GPa}$ for the neat epoxy polymer to $3.68 \mathrm{GPa}$ for the composite containing $17.2 \mathrm{vol} \%$ (volume fraction $V_{f}=$ 0.172 ) nanosilica. The tensile strength $\sigma_{m}$ remained relatively constant, although the results show that the strength may have been slightly improved at the highest silica contents. The tensile strain at break $\varepsilon_{b}$, on the other hand, is significantly reduced by the addition of the silica nanoparticles. All specimens broke without yield being observed. The DMA also showed a linear increase in the elastic modulus, that is, for the storage modulus $E^{\prime}$ with increasing silica content. The glass transition temperature $T_{g}$ remained almost constant at around $186^{\circ} \mathrm{C}$, and a small decrease in $T_{g}$ was observed only for the composite containing the highest amount of silica.

3.4.2. Anhydride-Cured System. The results from the tensile testing and the DMA of the neat anhydride-cured epoxy and its silica/epoxy nanocomposites are shown in Table 2 . The elastic modulus $E_{t}$ increased linearly from $3.03 \mathrm{GPa}$ for the neat epoxy polymer, to $4.15 \mathrm{GPa}$ for the composite containing $12.3 \mathrm{vol} \%$ silica (volume fraction $V_{f}=0.123$ ). There was also a gradual increase in the tensile strength $\sigma_{m}$, whereas a minor reduction in the strain at break $\varepsilon_{b}$ was observed.

A linear increase for the storage modulus $E^{\prime}$ was also observed. However, it is noteworthy that for the anhydridecured system there was also a linear decrease in $T_{g}$ with increasing silica content. The decrease in $T_{g}$ was from $155^{\circ} \mathrm{C}$ 
TABLE 1: Material properties of the amine-cured epoxy polymer and silica/epoxy nanocomposites, obtained from tensile testing and DMA testing.

\begin{tabular}{lccccc}
\hline \multicolumn{2}{c}{ Silica content } & Tensile testing & DMA & $\varepsilon_{b}$ & $E^{\prime}$ \\
wt\% & vol\% & $\begin{array}{c}E_{t} \\
(\mathrm{GPa})\end{array}$ & $\begin{array}{c}\sigma_{m} \\
(\mathrm{MPa})\end{array}$ & $(\%)$ & $\begin{array}{c}T_{g} \\
(\mathrm{GPa})\end{array}$ \\
\hline 0.0 & 0.0 & $2.61 \pm 0.03$ & $69 \pm 4$ & $4.8 \pm 0.6$ & $2.61 \pm 0.03$ \\
6.6 & 3.7 & $2.80 \pm 0.05$ & $68 \pm 2$ & $4.1 \pm 0.2$ & $2.87 \pm 0.05$ \\
13.4 & 7.8 & $3.04 \pm 0.07$ & $66 \pm 2$ & $3.5 \pm 0.3$ & $3.09 \pm 0.03$ \\
20.3 & 12.3 & $3.31 \pm 0.08$ & $69 \pm 1$ & $3.3 \pm 0.2$ & $3.37 \pm 0.06$ \\
27.5 & 17.2 & $3.68 \pm 0.11$ & $67 \pm 4$ & $2.7 \pm 0.3$ & $3.70 \pm 0.05$ \\
\hline
\end{tabular}

TABLE 2: Material properties of the anhydride-cured epoxy polymer and silica/epoxy nanocomposites, obtained from tensile testing and DMA testing.

\begin{tabular}{|c|c|c|c|c|c|c|}
\hline \multicolumn{2}{|c|}{ Silica content } & \multicolumn{3}{|c|}{ Tensile testing } & \multicolumn{2}{|c|}{ DMA } \\
\hline wt $\%$ & vol\% & $\begin{array}{c}E_{t} \\
(\mathrm{GPa})\end{array}$ & $\begin{array}{c}\sigma_{m} \\
(\mathrm{MPa})\end{array}$ & $\begin{array}{c}\varepsilon_{b} \\
(\%)\end{array}$ & $\begin{array}{c}E^{\prime} \\
(\mathrm{GPa})\end{array}$ & $\begin{array}{c}T_{g} \\
\left({ }^{\circ} \mathrm{C}\right)\end{array}$ \\
\hline 0.0 & 0.0 & $3.03 \pm 0.07$ & $85 \pm 1$ & $5.8 \pm 0.5$ & $2.99 \pm 0.04$ & $155 \pm 0$ \\
\hline 4.4 & 2.5 & $3.28 \pm 0.05$ & $89 \pm 1$ & $5.7 \pm 0.4$ & $3.24 \pm 0.04$ & $154 \pm 1$ \\
\hline 9.1 & 5.4 & $3.53 \pm 0.10$ & $89 \pm 2$ & $4.9 \pm 0.7$ & $3.41 \pm 0.08$ & $151 \pm 0$ \\
\hline 14.2 & 8.6 & $3.80 \pm 0.09$ & $88 \pm 5$ & $4.3 \pm 1.1$ & $3.75 \pm 0.06$ & $146 \pm 1$ \\
\hline 19.8 & 12.3 & $4.15 \pm 0.08$ & $93 \pm 0$ & $5.1 \pm 0.3$ & $3.97 \pm 0.10$ & $143 \pm 1$ \\
\hline
\end{tabular}

to $143^{\circ} \mathrm{C}$. It has been shown in the literature that there may be a correlation between the observed $T_{g}$ and the elastic modulus for neat polymer, for example [49]. In our case, by varying the resin/hardener ratio of the neat anhydridecured epoxy polymer, we have observed by DMA that a reduction in $T_{g}$ of $10^{\circ} \mathrm{C}$ may coincide with an increase of the elastic modulus by $0.15 \mathrm{GPa}$ when there is an excess of hardener, resulting in different crosslink densities of the three-dimensional polymer network. The increased elastic modulus may be explained in terms of free volume, that is, a higher packing of the polymer chains and higher material density as the result of lower crosslink density [10, 49]. For the anhydride-cured nanosilica/epoxy system, this may indicate that nonstoichiometric amounts of hardener have also been used when producing the nanocomposite plates. In addition, this observation indicates that also the state of the epoxy matrix may contribute partly to the elastic modulus of the nanocomposites. A variation in $T_{g}$ as a result of the presence of silica has also been observed and reported by others $[6,50]$.

\section{Model Parameters}

In the next sections, the flexibility, accuracy, and main areas of application for the models described above are discussed. This is done by comparing the model calculations with experimental results for some relevant nanocomposites. Polymer matrix nanocomposites with two different inclusions are then considered: (1) spherical particles (Section 5) and (2) randomly distributed fiber-like particles (Section 6).

Before comparing the model calculations with the experimental results for different composites, the general and system independent model parameters used to describe the stiffness properties of the different nanocomposites are considered.
4.1. Properties of the Interphase Region. As described for the interphase model by Odegard et al. in Section 2.3.3, the interphase is defined as the region surrounding the particles, with different elastic properties compared to the bulk matrix. As shown in Figure 1, we assume that the interphase has a constant thickness $t_{i}$ and that the radius of the interphase $r_{i}$ is expressed as

$$
r_{i}=n r_{p}
$$

The dimensionless parameter $n$ is here denoted as the interphase thickness factor. As also pointed out above, the elastic properties are assumed to be constant through the interphase.

The thickness of the interphase is included in the stiffness calculations through the expressions for the volume fractions. For the case of spherical particles, the volume of the particles is given by $v_{p}=4 / 3 \pi r_{p}^{3}$. From this, the volume of the interphase can be calculated as

$$
\begin{aligned}
v_{i} & =\frac{4}{3} \pi\left(n r_{p}\right)^{3}-\frac{4}{3} \pi r_{p}^{3}=\frac{4}{3} \pi r_{p}^{3}\left(n^{3}-1\right) \\
& =\left(n^{3}-1\right) v_{p} .
\end{aligned}
$$

Hence, the volume of the interphase is $\left(n^{3}-1\right)$ times the volume of the particle. As a consequence of this, the volume fraction of the interphase is $\left(n^{3}-1\right)$ times the volume fraction of the particles; that is, $V_{i}=\left(n^{3}-1\right) V_{p}$. Moreover, the volume fraction of the matrix phase is given as $V_{m}=1-\left(n^{3}-1\right) V_{p}-$ $V_{p}=1-n^{3} V_{p}$. For other spheroidal shapes we end up with the same expressions for the volumes and volume fractions; see [10] for the special case of volume fractions for carbon nanotubes in a matrix system. 


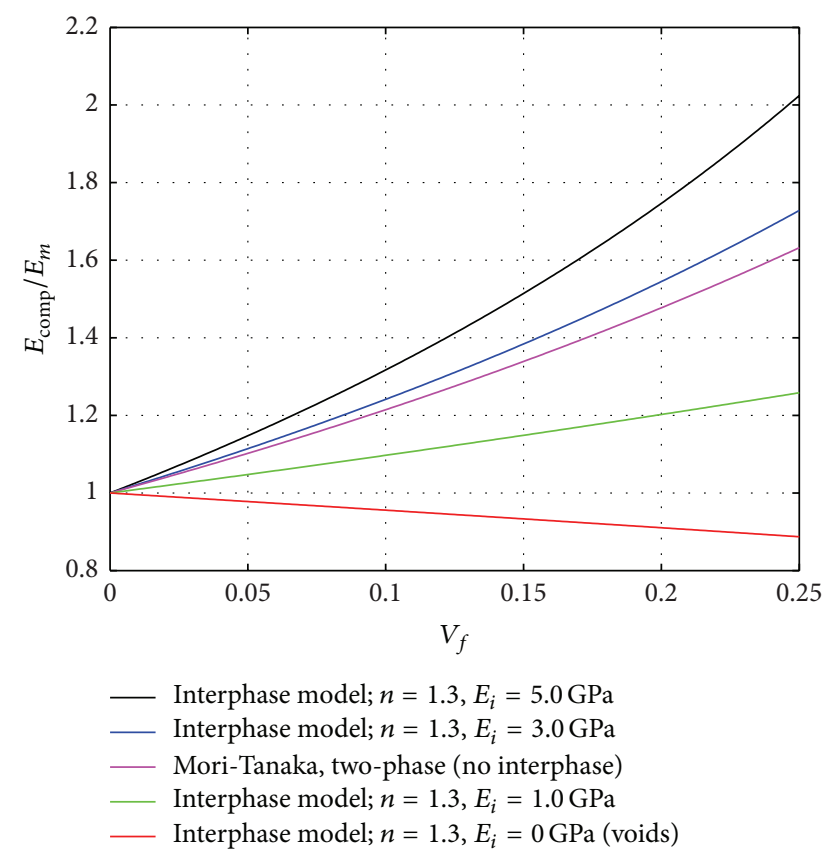

Figure 2: Model results for the silica/epoxy composite elastic stiffness. The interphase thickness factor is set to a constant value of 1.3, and the interphase elastic stiffness is varied.

To better explain the effect on the two key model parameters for the interphase (i.e., the interphase thickness factor and the interphase elastic stiffness), we now assume a nanocomposite where the bulk matrix has an elastic stiffness of $2.5 \mathrm{GPa}$ and a Poisson's ratio of 0.35 , with a small volume fraction of spherical nanoparticles with an elastic stiffness of $70 \mathrm{GPa}$ (28 times the bulk matrix) and a Poisson's ratio of 0.2. The interphase model is applied in the calculations.

First, the interphase thickness factor is set to a constant value, say 1.3 , and the interphase elastic stiffness is varied. An interphase elastic stiffness equal to the bulk matrix stiffness, which is the two-phase Mori-Tanaka model case, is in this case our reference. (Note that an elastic interphase stiffness equal to the bulk matrix stiffness results in inversion of a singular matrix, and, hence, this particular case is outside the area of application for the interphase model.) As can be observed in Figure 2, increasing the elastic stiffness of the interphase region will increase the stiffness of the composite, whereas a reduction of the same stiffness parameter will reduce the composite stiffness. When approaching the case of no interphase elastic stiffness, that is, a polymer with voids, the calculated composite stiffness is reduced to a value below the bulk stiffness.

Second, the stiffness of the interphase is set to a constant value, and the interphase thickness factor is varied. The case $n=1.0$, that is, no interphase region, is now our reference, and in this case the composite stiffness overlaps the composite stiffness calculated using the two-phase Mori-Tanaka model. If the interphase elastic stiffness is set to $4 \mathrm{GPa}$, which is a bit higher than the bulk matrix stiffness, but still much lower than the particle inclusion stiffness, from Figure 3 we observe

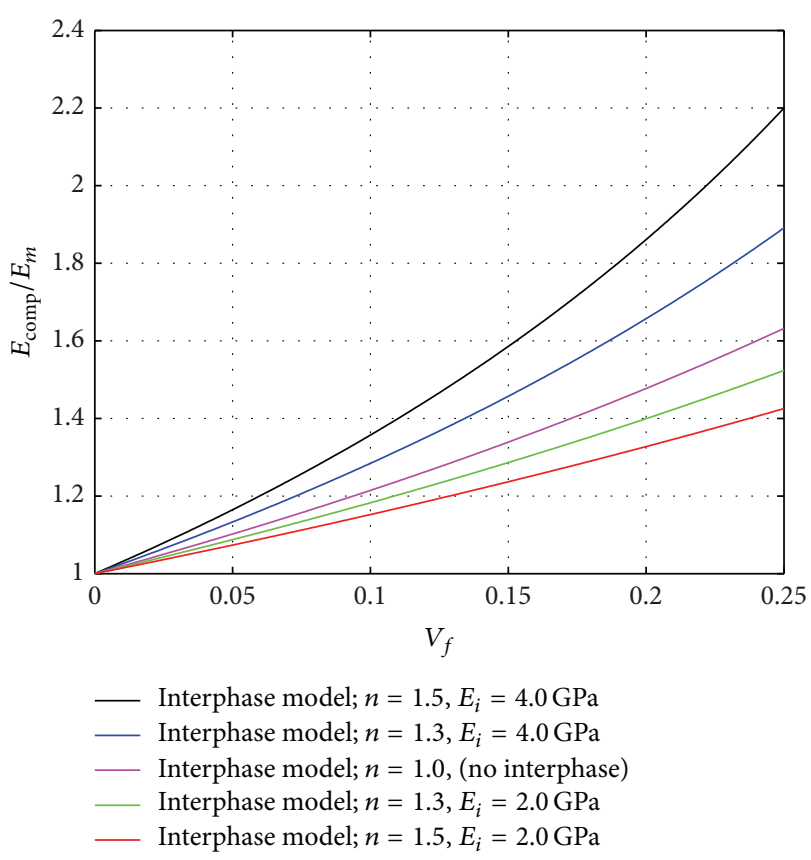

FIGURE 3: Model results for the silica/epoxy composite elastic stiffness. The interphase thickness factor and the interphase elastic stiffness are varied.

that the composite stiffness is increased with increasing $n$. With a constant interphase elastic stiffness of $2 \mathrm{GPa}$, which is a bit lower than the bulk matrix stiffness, the composite stiffness is shown to be reduced for increasing $n$. This seems like a logical behavior and confirms that the model provides the expected trend.

In the examples above, no precise estimate was given for the interphase elastic stiffness, and no restrictions were made for the interphase thickness factor. This will certainly vary for different nanocomposites, but some conclusions can be made. For the model calculations to actually match the stiffness improvement observed experimentally, the interphase elastic stiffness should be higher than the neat matrix stiffness. Also, taking into account the fact that the interphase volume fraction is $\left(n^{3}-1\right)$ times the volume fraction of the particles, the interparticle distance sets some restrictions on $n$ [51-53].

For the interparticle distance, two idealized particle packing structures for the case of spherical particles, obtained from simple geometrical considerations taking the interphase into account, are shown in Figure 4. A contour map indicates the minimum distance between layers for each packing, where a negative value indicates particle interphase overlap (which is outside the area of application for the model). In case of hexagonal packing of the particles (Figure 4(a)), the interphase thickness factor should be less than 1.9 for particle volume fractions up to 0.10 . In case of simple cubic packing of the particles (Figure 4(b)), the interphase thickness factor should be less than 1.75 for particle volume fractions up to 0.10 . In a real situation, something between the two idealized packing structures would be expected. An equivalent plot has also been shown by Karger-Kocsis and Zhang [53] for 


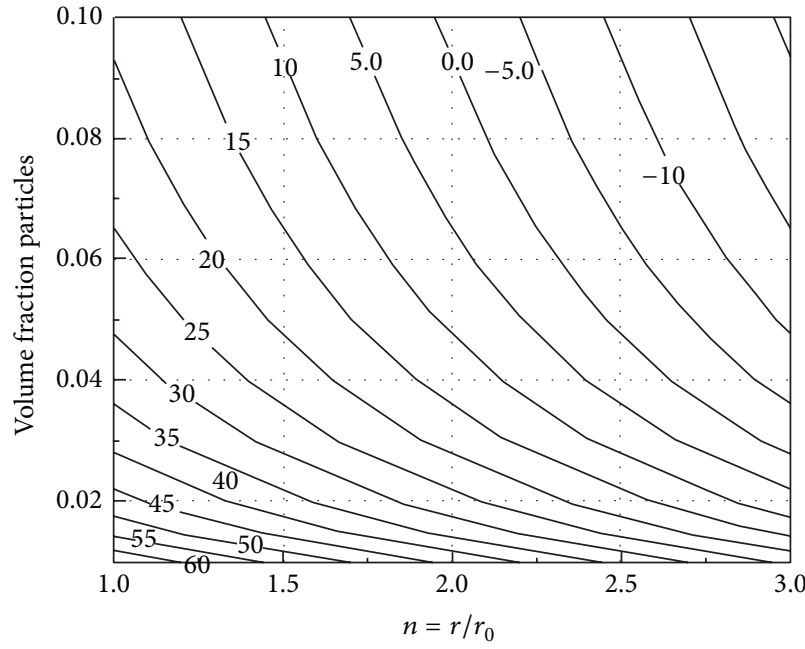

(a)

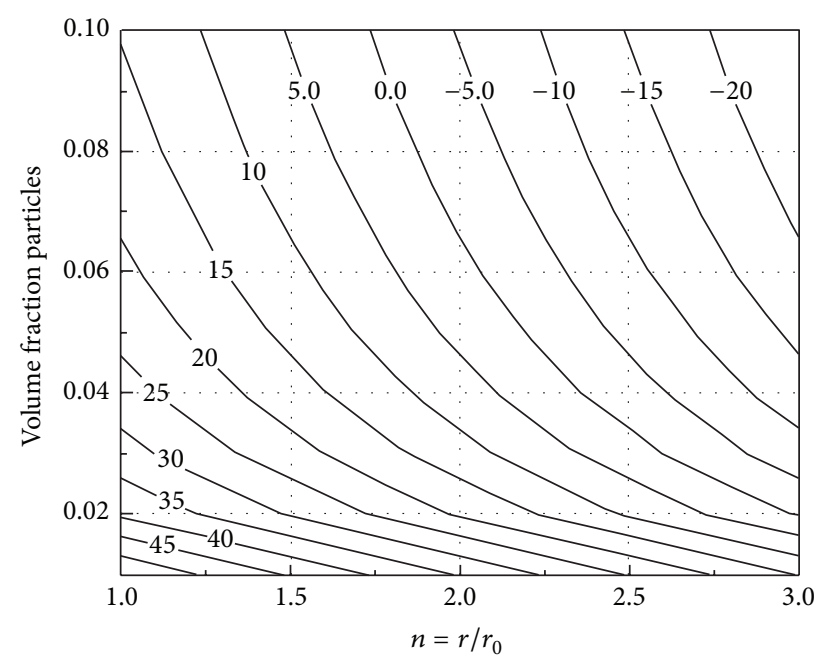

(b)

FIGURE 4: Packing structures of spherical particles in 3D. (a) Hexagonal packing; (b) simple cubic packing.

cubic distribution of spherical particles, for different particle diameters.

4.2. Degree of Exfoliation in Agglomerated Composites. For nanocomposites with fiber-like particle inclusions, it is likely that the particles are not fully dispersed and that a second inclusion phase is present in the form of particle agglomerates. In addition, one also needs to take into account the particle orientation distribution; without an enforced flow field as part of the production process, the particles are likely to be randomly oriented. The random orientation is taken care of by the models described above, by integrating over all orientations.

To quantify the amount of agglomerates and the volume fraction of particles inside the agglomerates, the twoparameter model by Shi et al. [41, 42] can be applied. Alternatively, the volume fractions of the dispersed (i.e., free) particles and agglomerates can be estimated using the degree of exfoliation (DOE) $\varphi_{\mathrm{DOE}}$, which can be expressed as

$$
\varphi_{\mathrm{DOE}}=\frac{V_{p, f}}{V_{p}},
$$

where $V_{p, f}$ is the volume fraction of free particles and $V_{p}$ is the total volume fraction of particles in the composite. In this latter case, $\varphi_{\mathrm{DOE}}$ can also be expressed by the volume fraction of the particles within the agglomerates of the composite. The DOE for a given nanocomposite can be estimated from a particle size analysis; see [55] for the case of dispersion of multiwall CNTs (MWCNTs) in a liquid solution. The DOE will vary as a function of volume fraction of $V_{p}$.

\section{Model Results for Spherical Particle Inclusions}

Three different nanosilica/epoxy composites are compared with the mathematical models in the following, including the elastic stiffness results of the amine-cured and anhydridecured composites given in Tables 1 and 2 . For these systems, a perfect dispersion of the spherical silica particles is assumed, and, hence, a two-phase nanocomposite is considered. The stiffness can in this case be estimated from the Halpin-Tsai equation (with $\beta=1.0$ ), the two-phase Mori-Tanaka model, and the interphase model. A Poisson's ratio of 0.35 is assumed for the neat epoxy polymers, as well as for the interphase (in the interphase model).

5.1. Results. First, for the amine-cured composite, the three different models are plotted together with the experimental data in Figure 5(a). To try to fit the interphase model results to the experimental data, the interphase thickness factor as a function of silica volume fraction was selected in the range from 1.05 to 1.1; see Figure 5(b). The elastic stiffness of the interphase is in this case set to a constant value of $4.0 \mathrm{GPa}$. As can be observed, the Halpin-Tsai equation overestimates the elastic stiffness of the composite. However, both the MoriTanaka two-phase model and the interphase model estimate the elastic stiffness very well for all volume fractions of silica (provided the selected interphase characteristics are employed for the interphase model). For higher volume fractions, the interphase model with a small interphase thickness resulted in best agreement with the experimental results.

Second, the modeling and experimental results for the anhydride-cured composite are given in Figure 6(a). In this case, again to try to fit with the experimental data, the interphase thickness factor was set much higher and varied between 1.7 and 1.4 as a function of the silica volume fraction; see Figure 6(b). The elastic stiffness of the interphase is also in this case set to a constant value of $4.0 \mathrm{GPa}$. As can be seen in the figure, the Halpin-Tsai equation and the interphase model estimate the elastic stiffness of the composite very well. The two-phase Mori-Tanaka model, on the other hand, underestimates the elastic stiffness of the composite. 


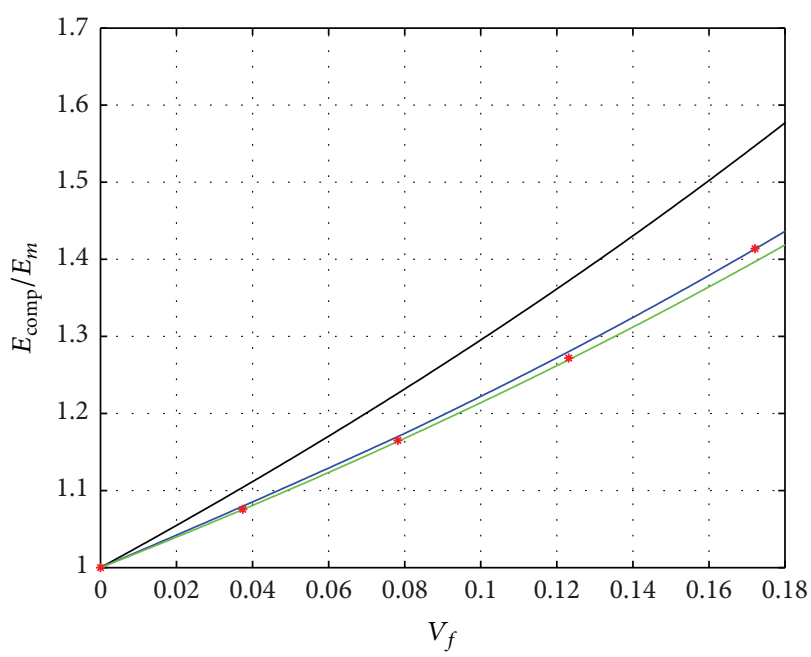

Halpin-Tsai

— Interphase, varying thickness

_ Mori-Tanaka, two-phase

* Experimental, amine-cured system

(a)

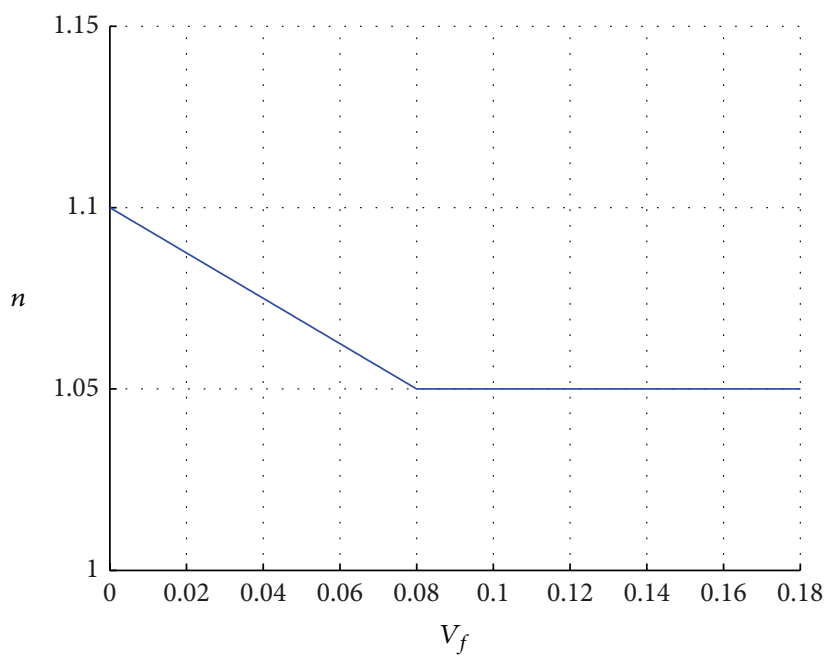

(b)

FIGURE 5: Model results and experimental data for the amine-cured nanosilica/epoxy system. (a) Composite elastic stiffness as a function of particle volume fraction; (b) interphase thickness factor.

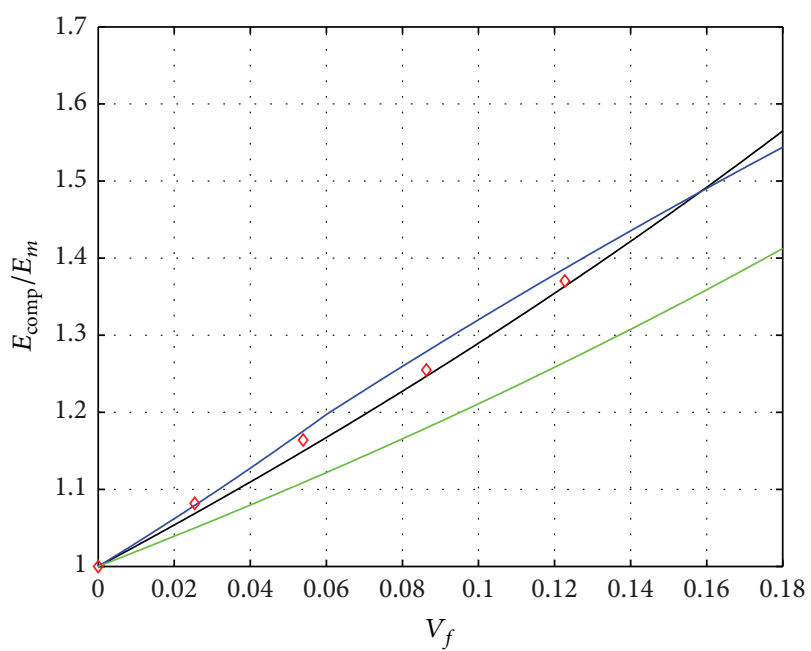

Halpin-Tsai

Interphase, varying thickness

- Mori-Tanaka, two-phase

$\diamond \quad$ Experimental, anhydride-cured system

(a)

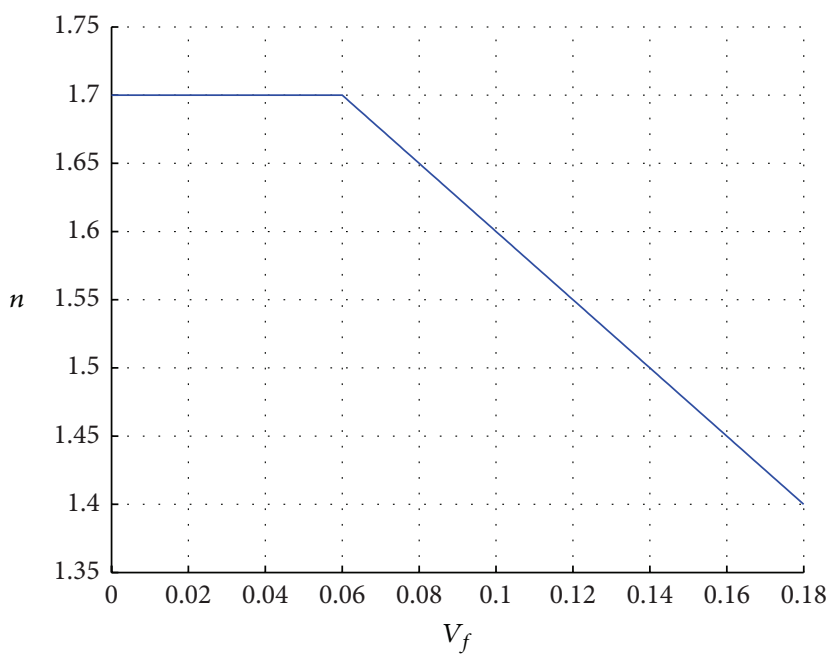

(b)

FIGURE 6: Model results and experimental data for the anhydride-cured nanosilica/epoxy system. (a) Composite elastic stiffness as a function of particle volume fraction; (b) interphase thickness factor.

Comparing the above two systems, a much lower interphase thickness factor was selected for the amine-cured composite. Therefore, the effect of selecting a lower and constant interphase thickness factor of 1.3 and varying the interphase elastic stiffness of the anhydride-cured composite is a relevant case. This is shown in Figure 7. It can be seen that an interphase stiffness between 5.0 and $6.0 \mathrm{GPa}$ agrees well with the experimental data. An elastic stiffness of $4.0 \mathrm{GPa}$ 


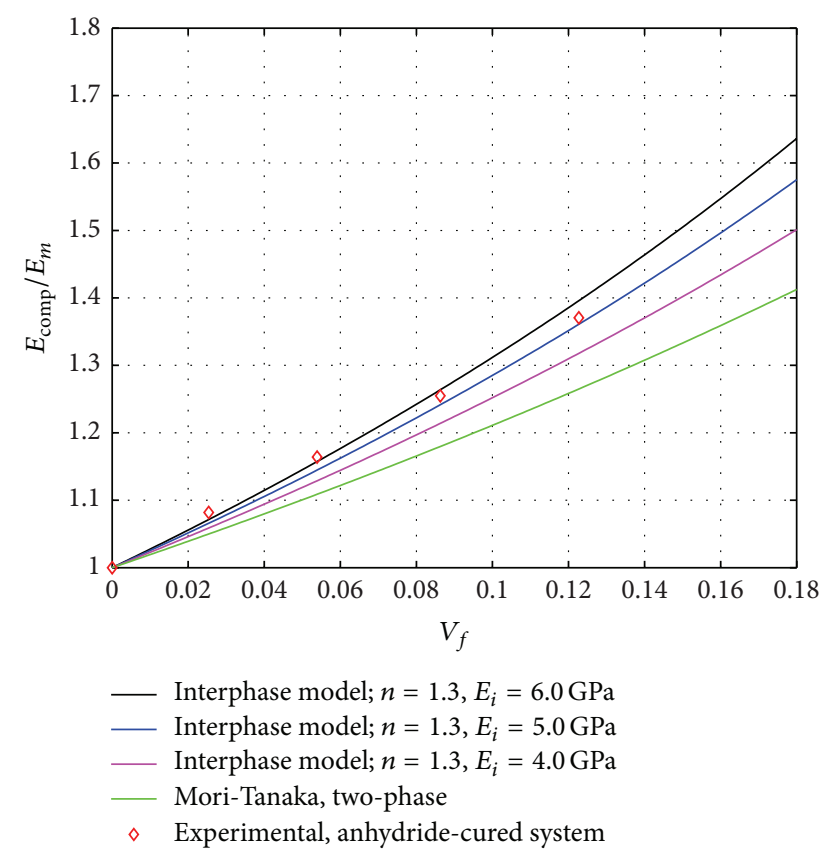

FIGURE 7: Model results and experimental data for the anhydridecured nanosilica/epoxy system. The interphase thickness factor is set to a constant value of 1.3 , and the interphase elastic stiffness is varied.

is observed to underestimate the composite stiffness. The two-phase Mori-Tanaka calculations are unchanged from the previous case and only included here for comparison.

Third, Johnsen et al. [54] reported results for a similar nanosilica/epoxy system. They used the same nanosilica particles, but the polymer was cured with a different anhydride hardener. Their measured composite elastic stiffness was compared to model calculations using the Halpin-Tsai equation, as well as the Lewis-Nielsen model for both no-slip $\left(k_{E}=2.167\right)$ and interfacial slippage $\left(k_{E}=0.837\right)$ conditions. A perfect dispersion of the nanoparticles was assumed. The experimental results and the calculated composite elastic stiffness are shown in Figure 8(a) [54]. In the figure, the results shown have been corrected to a silica density of $\rho_{p}=$ $2100 \mathrm{~kg} / \mathrm{m}^{3}$, which is identical to the density used in the above cases; the density applied by Johnsen et al. was $\rho_{p}=$ $1800 \mathrm{~kg} / \mathrm{m}^{3}$. The different silica density will affect the silica volume fraction, which again will slightly alter the slope of the elasticity curve. As can be observed in the plot, the Halpin-Tsai equation and the Lewis-Nielsen model with noslip interface condition give the same stiffness increase for increasing volume fractions. The two models underestimate the stiffness for low volume fractions and overestimate the stiffness for higher volume fractions. The two-phase MoriTanaka model and the Lewis-Nielsen model with slippage at the interface underestimate the elastic stiffness for all volume fractions. On the other hand, the calculated elastic stiffness using the interphase model can be tuned (as was done for the above two nanocomposite systems), such that there is an agreement with the experimental results for both low and high volume fractions. The elastic stiffness of the interphase is in this case set to $5.0 \mathrm{GPa}$, whereas the interphase thickness factor is varying between 1.6 and 1.2, as shown in Figure 8(b). With a reduced stiffness in the interphase region (i.e., $E_{i}<$ $\left.E_{m}\right)$ and constant interphase thickness factor for all volume fractions, the interphase model would have given a similar curve to that of the Lewis-Nielsen model with the "slippage" condition.

In the previous case, the thickness of the interphase for low volume fractions was set to a very large value, which may be unphysical and not according to measurements (e.g., [38]). In the same way as for the anhydride-cured system above, the interphase thickness for the nanosilica/epoxy system from Johnsen et al. is therefore reduced and set to a constant value of 1.3, whereas the interphase elastic stiffness is varied. As can be seen in Figure 9 [54], an interphase stiffness $E_{i}=$ 9.0 GPa agrees well with the available experimental data for low volume fractions. Similarly, an interphase stiffness of $E_{i}=4.0 \mathrm{GPa}$ agrees well with the experimental data for higher volume fractions. The Mori-Tanaka model is included for comparison.

5.2. Discussion. In the results presented above, the extent of the interphase was varied significantly in order to fit the experimental data. It can be argued whether the (selected) values for the interphase thickness factor $n$ are correct or not. However, some data can be found in the literature regarding the extent of the interphase around nanoparticles. According to Antonelli et al. [56], the extent of the interphase was $3 \mathrm{~nm}$ for a silica/epoxy system with $17 \mathrm{~nm}$ silica particles. For the silica particles used here, this gives a value of $n=1.15$. Holt et al. [38] studied a silica/poly(2-vinylpyridine) system. They found that the interphase thickness was $4-6 \mathrm{~nm}$ for silica particles with a diameter of $30 \mathrm{~nm}$ and that it was independent of particle concentration (i.e., particle volume fraction). For $20 \mathrm{~nm}$ particles, this results in a value of $n$ in the range from 1.2 to 1.3. Thus, these literature values and the values used in the interphase model are of the same order, although the highest values may be considered unrealistically high. Interphase layer thicknesses of $10 \mathrm{~nm}$, here resulting in a value of $n$ of 1.5, have, however, been reported; see [56] and the references therein.

The two silica/epoxy systems that were tested here (in Section 3) gave different values for the elastic stiffness of the neat epoxy polymer. It follows that the relative increase in elastic stiffness was also different. On the whole, the elastic stiffness was increased relatively more for the anhydridecured system with the addition of silica, compared to the amine-cured system. The difference between the two systems is likely to be explained by chemical and physical differences, such as the difference in the polymer network structure, as well as differences in interfacial binding properties between the particles and the polymer. As already discussed in Section 3.4.2, $T_{g}$ of the anhydride-cured system decreases for increasing volume fractions. The same behavior was not observed for the amine-cured system. In addition, DMA measurements of the neat anhydride-cured epoxy polymer showed that the elastic stiffness could be increased when $T_{g}$ was decreased as a result of nonstoichiometric resin/hardener 


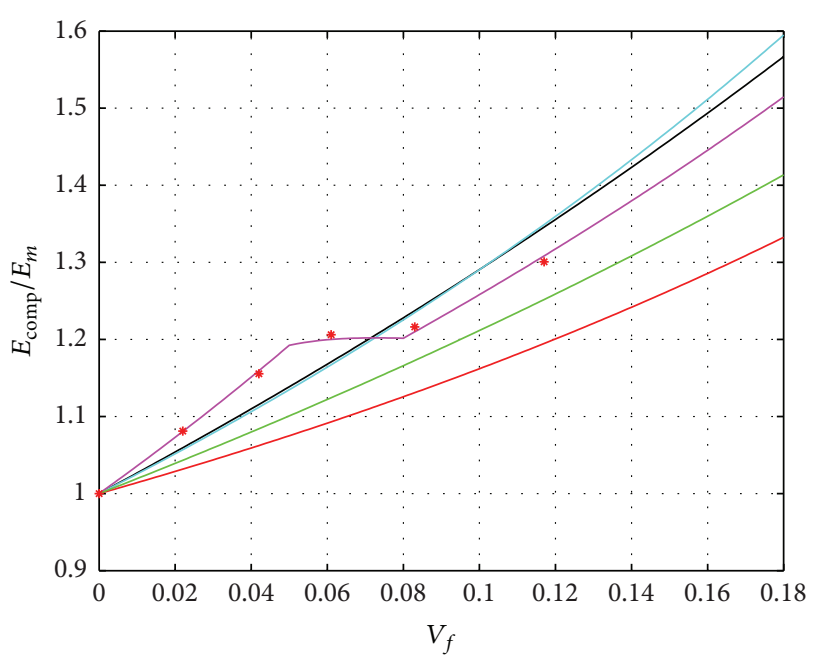

Halpin-Tsai
Nielsen-no-slip
Nielsen-slip
Interphase, varying thickness
- Mori-Tanaka, two-phase
Experimental, Johnsen et al., $2007, \rho=2100 \mathrm{~kg} / \mathrm{m}^{3}$

(a)

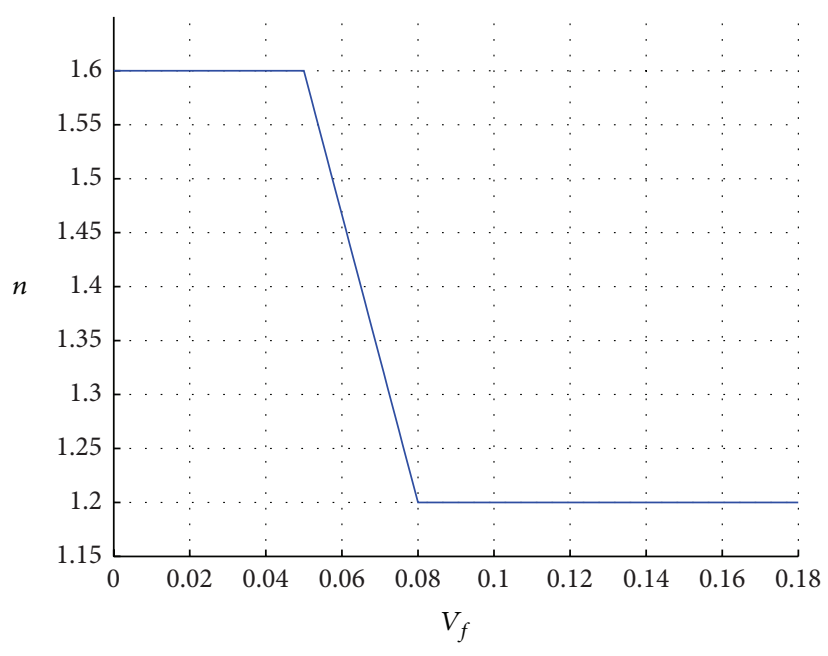

(b)

Figure 8: Model results and experimental data for the nanosilica/epoxy system in Johnsen et al. [54]. (a) Composite elastic stiffness as a function of particle volume fraction; (b) interphase thickness factor.

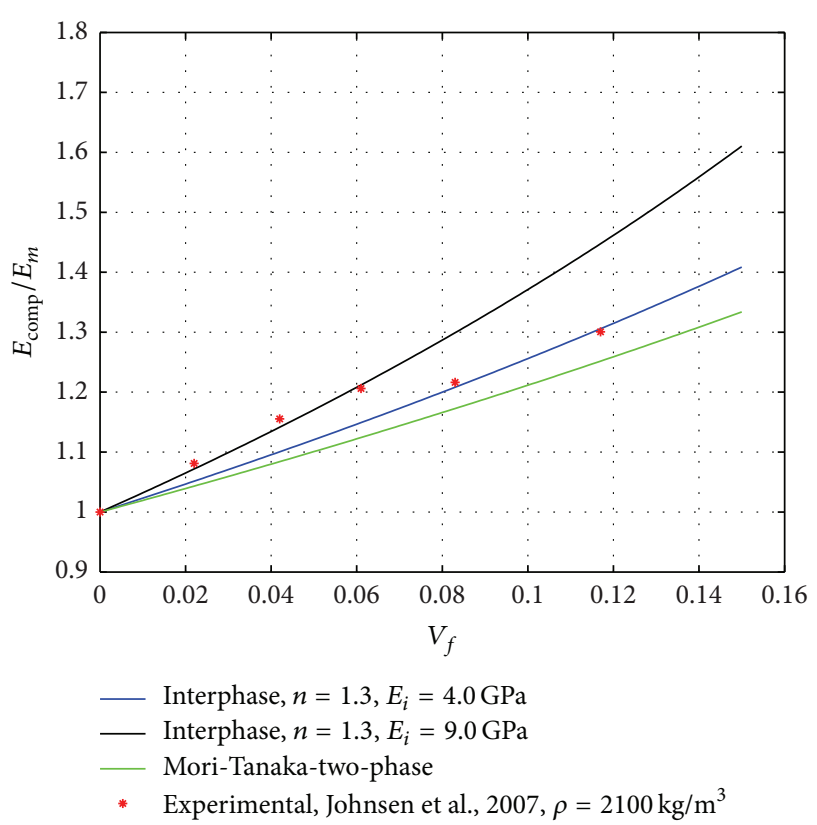

FIGURE 9: Model results and experimental data taken from Johnsen et al. [54]. Composite elastic stiffness of nanosilica/epoxy composite as a function of volume fraction. The interphase thickness factor and the interphase elastic stiffness are varied for the interphase model.

ratios (the elastic stiffness increase was around $0.15 \mathrm{GPa}$ when $T_{g}$ was decreased by $10^{\circ} \mathrm{C}$ ). Since $T_{g}$ of the anhydride-cured composite is decreased, it could mean that the elastic stiffness is also influenced by an altered polymer structure. Hence, the effective increase in elastic stiffness, solely due to the addition of silica, may not be as high as that indicated by the experimental results.

When comparing the experimental results with the mathematical models, it can be presumed that the properties of the interphase region surrounding the particles are different for the amine-cured system and the anhydride-cured system. For the amine-cured system, the two-phase Mori-Tanaka model was found to agree well with the experimental results for all volume fractions. For the interphase model, only a small interphase thickness was required to obtain good agreement with the experimental results. This indicates that the molecular structure of the polymer network is not significantly influenced by the particle inclusions. This is also indicated by the stable $T_{g}$ values. Moreover, the Halpin-Tsai equation was found to overestimate the stiffness and it did not describe the stiffness properties of the amine-cured system very well, even though a two-phase composite with no-slip condition at the interface between the particle and the polymer is assumed.

For the anhydride system, on the other hand, the stiffness calculated using the Halpin-Tsai equation agrees well with the experimental data, whereas the Mori-Tanaka model underestimated the elastic stiffness. The interphase model was also found to agree well, when assuming an interphase region, with a slightly higher elastic stiffness compared to the bulk matrix. This observation, combined with the observation of the reduction in $T_{g}$ of the composites, indicates that the polymer network is altered due to the particle inclusions (or possibly also by the mixing ratio of the different components). 
Mathematically it should then be plausible to model the stiffness increase by an interphase region with different elastic properties compared to the nonreinforced neat matrix. Since the two-phase Mori-Tanaka model does not include this additional stiffness region, it is predictable that the model underestimates the composite stiffness. However, the calculated stiffness from employing the Halpin-Tsai equation agrees well with the experimental results, even if the properties of the composite are different from what is assumed in the model. The reason for this is at present unknown.

For the system presented by Johnsen et al. [54], the silica particles were assumed to be fully dispersed in the polymer matrix, even for higher concentrations, and $T_{g}$ of the composite was relatively stable. Thus, the system should be considered as a two-phase system with no significant interphase region surrounding the particles. As reported above, the Halpin-Tsai equation and the Lewis-Nielsen model with no-slip interface condition do not agree very well with the experimental results in this case but provide some kind of average stiffness estimate for all particle concentrations. The Mori-Tanaka model underestimates the stiffness for low volume fractions. For higher volume fractions, on the other hand, there is good agreement. Again, for the interphase model it is possible to vary the model parameters, so that the stiffness is in agreement with the experimental values for all volume fractions included. A plausible explanation is that the elastic properties, in fact, vary as a function of volume fraction, which then can be modeled either by an increased interphase region or by an increase in the elastic stiffness of this region. Finally, the Lewis-Nielsen model with the slippage interface conditions underestimates the elastic stiffness for all volume fractions and, hence, does not seem to mimic the interface conditions in an appropriate way, even though $k_{E}$ is adjusted to fit the system considered.

\section{Model Results for Fiber-Like Particle Inclusions}

Fiber-like particle inclusions in polymer systems may require other models than those used for spherical inclusions. First of all, the orientation of the particles needs to be accounted for. Moreover, the particles are often more challenging to disperse, resulting in a second inclusion phase in the form of agglomerates of the particles. Two different nanocomposite systems are considered: (1) a CNF/epoxy composite and (2) a MWCNT/polystyrene composite.

6.1. CNF/Epoxy Composite. A model for a CNF/epoxy composite with agglomerates has been presented by Gershon et al. [5]. The amount of free, randomly orientated CNFs was expressed by the degree of exfoliation (DOE), which is calculated from the volume fractions of $\mathrm{CNF}$ in the agglomerates and in the polymer matrix. Based on their DOE curve, Gershon et al. concluded that the modulus of $\mathrm{CNF}$ is $30 \mathrm{GPa}$, even though a value of one order higher was expected. Now, if we instead assume a stiffness value for the CNFs of one order higher, say, $200 \mathrm{GPa}$, the DOE values are only slightly changed; both sets of values are shown in
Figure 10(b). The latter values should therefore be equally representative as the reported values, at least for defining a continuous DOE function from the discrete values. Based on the above discussion, a stepwise linear DOE function is defined and applied for the composite considered and also included in the figure.

In this case, only the three-phase Mori-Tanaka model is applicable for comparison since the interphase model is restricted to one inclusion phase. For the CNF/epoxy system considered, the bulk elastic stiffness of the matrix is $1.95 \mathrm{GPa}$. The matrix Poisson's ratio is not given but set to 0.35 . Moreover, the CNFs are said to have a diameter of $100-200 \mathrm{~nm}$ and a length of $10-30 \mu \mathrm{m}$. The CNF aspect ratio is therefore set to 150 in our calculations. Furthermore, the elastic modulus of the CNFs is set to $200 \mathrm{GPa}$, and Poisson's ratio is set to 0.20 . Finally, for the agglomerates, the measured average elastic stiffness is $4.2 \mathrm{GPa}$ and Poisson's ratio is set to 0.35 , which is equal to the matrix.

The three-phase Mori-Tanaka model results together with the stiffness values from Gershon et al. are shown in Figure 10(a) [5]. As can be observed, the Mori-Tanaka model agrees very well with the Gershon et al. calculations. It should also be noted that a slightly higher DOE value for the higher volume fractions would have given an even better match for all volume fractions in the range. However, this would have required a much higher DOE than that calculated by Gershon et al.

6.2. MWCNT/Polystyrene Composite. In this second case, we consider a MWCNT/polystyrene composite. Experimental values are reported by Andrews et al. [57]. Only the polystyrene elastic stiffness is given in the paper; that is, $E_{m}=1.9 \mathrm{GPa}$ [57]. However, representative parameter values, which are needed for the modeling, can be found elsewhere in the literature. From the work by Shi et al. [41], who considered the same system, Poisson's ratio for polystyrene is given; $v_{m}=0.3$. For the MWCNTs, the values are taken from the work of $\mathrm{Lu}[58] ; E_{\mathrm{CNT}}=975 \mathrm{GPa}$ and $v_{\mathrm{CNT}}=0.27$. The aspect ratio of the MWCNTs is set to 1000 , based on the length and diameter range reported by Andrews et al. [57].

First, we investigate the applicability of the two-phase Mori-Tanaka model, the interphase model, and the modified Halpin-Tsai equation for randomly oriented fibers. The results are given in Figure 11 [57]. It can be seen that the model results agree with the calculated stiffness reported by Shi et al. [41, 42] for the same nanocomposite. Also, the Mori-Tanaka model and the interphase model with zero interphase thickness, that is, $n=1$, result in overlapping stiffness curves. (The interphase model curve is not shown in the plot.) This shows that the averaging strategy employed for the extended version of the interphase model should be valid. Moreover, an interphase thickness factor larger than one and a reduced stiffness of the interphase region do not reduce the composite stiffness significantly. Hence, both model approaches overestimate the composite elastic stiffness compared to the experimental data. This could partly be due to a less than perfect dispersion of the MWCNTs in the experiments conducted by Andrews et al. [57], that 


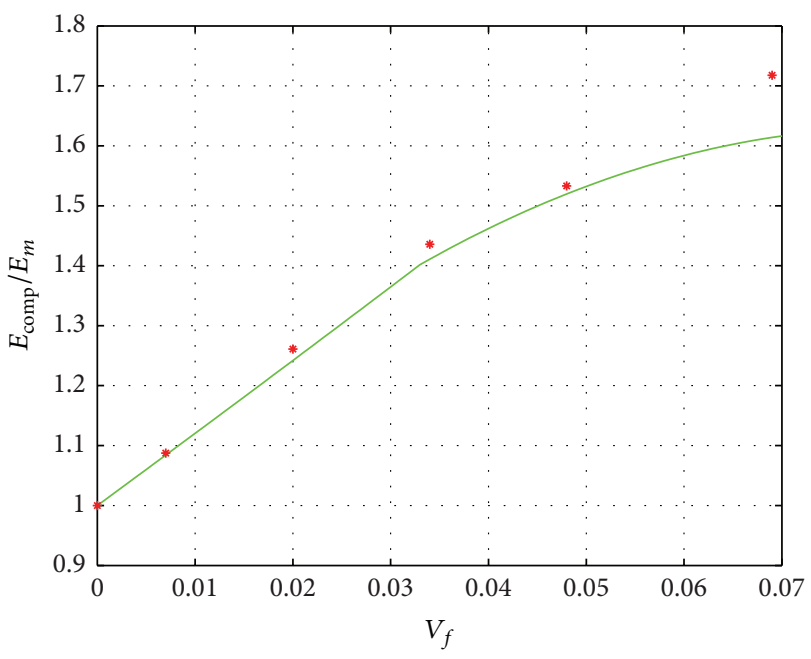

Mori-Tanaka, three-phase

* Gershon et al., 2010

(a)

Figure 10: Model results for the CNF/epoxy composite in Gershon et al. [5]. (a) Composite elastic stiffness as a function of particle volume fraction; (b) DOE curve for the CNF/epoxy.

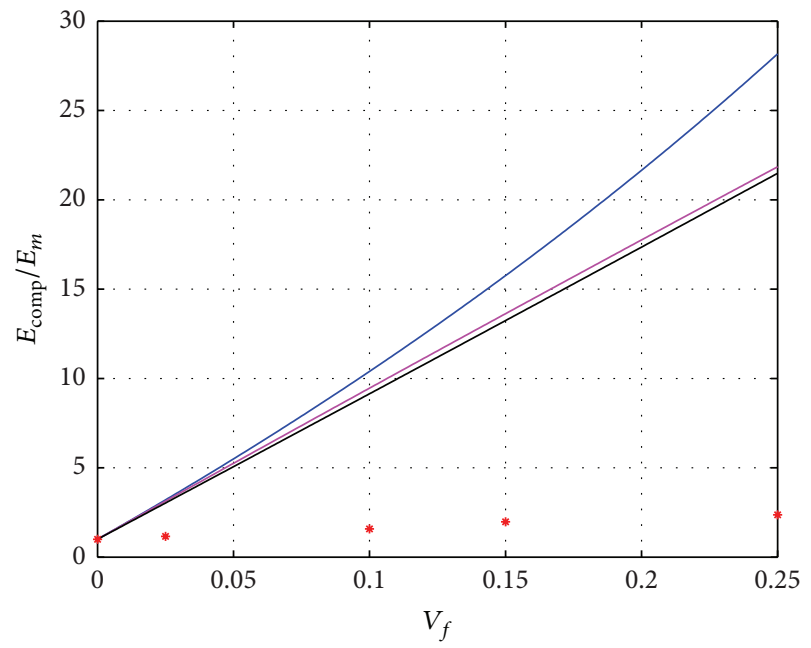

\footnotetext{
- Mori-Tanaka, two-phase

— Interphase model, $n=1.3, E_{i}=1.0 \mathrm{GPa}$

- Halpin-Tsai, randomly oriented fibers

* Experimental, Andrews et al., 2002
}

FIgURE 11: Model results and experimental data for the MWCNT/polystyrene composite in Andrews et al. [57]. Composite elastic stiffness as a function of particle volume fraction.

is, $\mathrm{DOE}<1$. Finally, the modified Halpin-Tsai equation also overestimates the elastic stiffness.

From the above case, other effects than the interphase effects seem to significantly affect the composite stiffness in this case. We therefore instead apply the three-phase MoriTanaka model. Figure 12 [57] displays the model results and

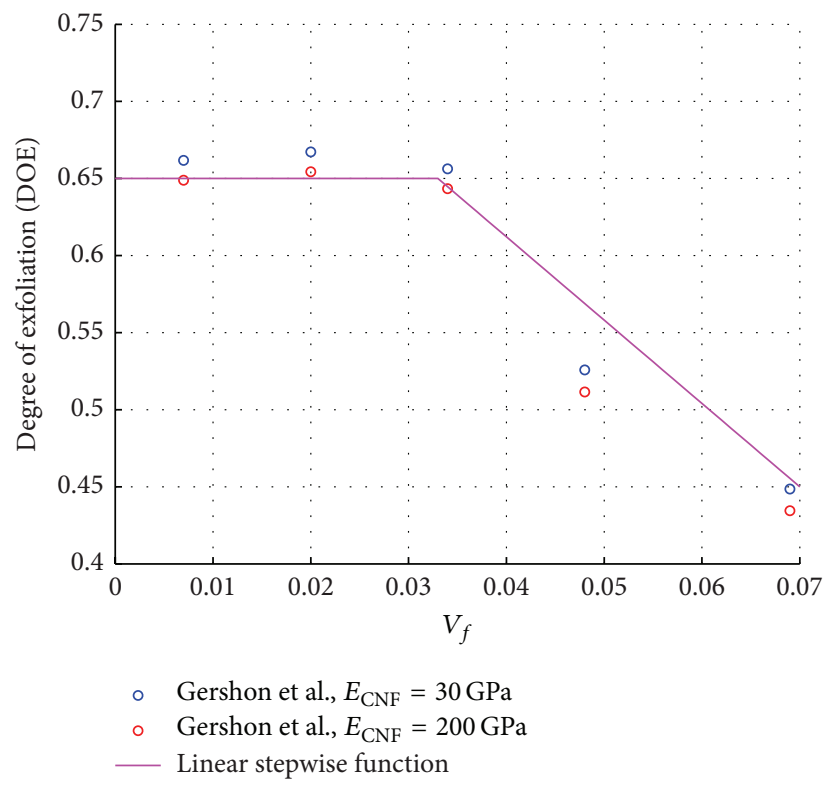

(b)

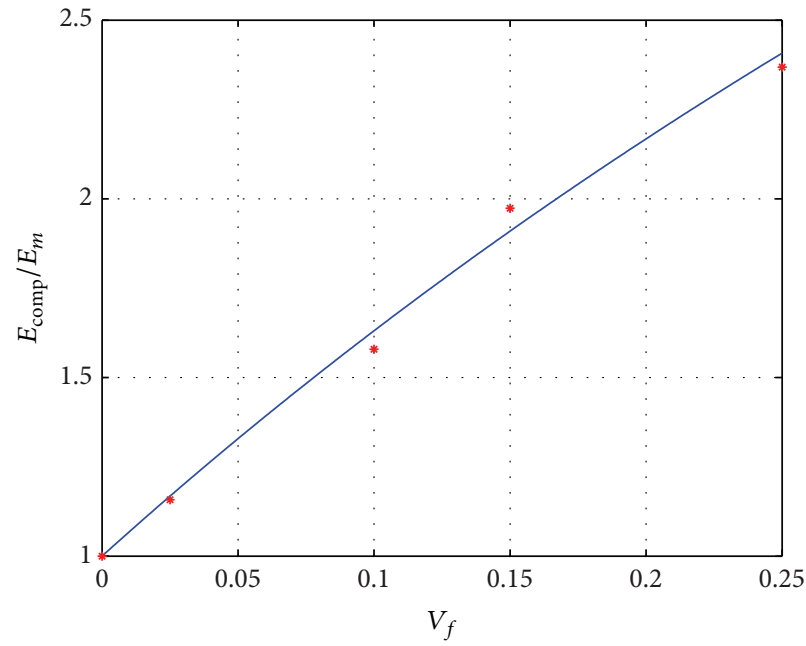

_ Mori-Tanaka, three-phase

* Experimental, Andrews et al., 2002

FIGURE 12: Model results and experimental data for the MWCNT/polystyrene composite in Andrews et al. [57]. Composite elastic stiffness as a function of particle volume fraction using the three-phase Mori-Tanaka model with voids.

the experimental data. To get agreement with the experimental results, the elastic stiffness of the agglomerated areas is set to zero, that is, assuming that the agglomerated MWCNTs do not contribute to the composite stiffness. Hence, the agglomerates are considered to be voids in the model. In this case, an assumed constant degree of exfoliation of 0.1 for all 
volume fractions gives good correlation between the model and the experimental results.

6.3. Discussion. The first observation from the study of the two nanocomposites with fiber-like inclusions is that significant stiffness improvements can be obtained if the inclusions with extreme mechanical properties and high aspect ratio are perfectly dispersed in the matrix. This conclusion seems to yield even for the case of reduced load transfer at the interphase. The second observation is that the composite stiffness is significantly reduced due to agglomerates of the particles. But even a small amount of dispersed particles, that is, a very low value of the degree of exfoliation, will give a stiffness increase.

Considering the models and the experimental data, only the three-phase Mori-Tanaka model is applicable and relevant for composites with agglomerates. For the results presented by Gershon et al. and shown in Section 6.1, the volume fraction of the CNFs included in the agglomerates was found to give a stiffness increase compared to the bulk matrix. For the results presented by Andrews et al. [57], the agglomerates, on the other hand, seem not to improve the composite stiffness, but rather being the main factor for the large stiffness reduction (compared to the perfect dispersion case).

For the MWCNT/polystyrene case, the large stiffness reduction due to the agglomerates may not be in accordance with the results reported by Andrews et al., where the structural imperfections and impurities for the as-received MWCNTs were said to be removed (by heating to graphitization temperatures) before making the MWCNT/polystyrene composite of the well-dispersed MWCNTs. The model results presented here may therefore give some new insight. Our calculations have shown that a well-dispersed composite with optimal load transfer may actually not be the case. One parameter that can be changed in the calculations, to help support the conclusions by Andrews et al., is the elastic stiffness of the MWCNTs. A stiffness reduction of the MWCNTs from $975 \mathrm{GPa}$ to $450 \mathrm{GPa}$ is found to dramatically reduce the composite stiffness. The stiffness does, however, not seem to be significantly affected by the variation in aspect ratio in the same way; a reduction of the aspect ratio from 1000 to 250 is not found to significantly reduce the composite stiffness.

\section{Summary and Conclusion}

In this paper, the main objective has been on identifying models that are relevant to include in a "model toolbox" for estimation of different nanocomposites with varying geometry and constituent materials with different properties. Different mathematical models are described for the elastic modulus of nanoparticle/epoxy systems. Agglomerates and particle interphase properties are two relevant factors for the elastic stiffness increase. A set of flexible models for nanocomposites have been described, which are relevant in multiscale modeling, including finite element method analyses, of advanced high-performance composite structures. Extensions of the models are also presented to include more flexibility in the choice of particle geometry and orientation.
The suggested models for modeling of the nanocomposites, that is, the multiphase Mori-Tanaka model and the Odegard et al. interphase model, are composite system independent models containing a few general model parameters with a clear physical meaning. For the interphase model, only two parameters are included: the interphase thickness factor and the interphase elastic stiffness.

For the three nanosilica/epoxy systems considered, the calculated stiffness using the Mori-Tanaka model and the interphase model was compared to the experimental results, as well as the results from using the Halpin-Tsai equation and the slippage model by Lewis and Nielsen. Due to differences in the applied polymer systems, different stiffness increases were obtained for the nanocomposites. From adjusting the model parameters, the interphase model was found to agree well for all three systems, both for low and high volume fractions. Moreover, the two-phase Mori-Tanaka model agreed well for systems where the interphase properties do not seem to be significant for the stiffness increase. The Halpin-Tsai equation was applicable for predicting the stiffness increase for the anhydride-cured system, but not for the two other composites. The Lewis-Nielsen model only seems to give some kind of average stiffness for all volume fractions.

The calculated stiffness increase from employing the interphase model, the Halpin-Tsai equation, and the general multiphase Mori-Tanaka model was also compared to experimental and model results for a CNF/epoxy composite and a MWCNT/polystyrene composite. For fiber-like particles, the interphase properties seem to be less important for the stiffness increase, compared to other factors. One reason may be that the fiber-like particles are entangled, resulting in agglomerates with less or no stiffness contribution. The threephase Mori-Tanaka model was found to agree well with the results for both nanocomposites considered.

As a final and overall conclusion, the multiphase MoriTanaka model and the Odegard et al. interphase model are found to be flexible, system independent, and applicable for calculating the elastic stiffness properties of the various nanocomposites, including both low and high volume fractions. These models are therefore preferred over more traditional models in estimation of the macroscopic elastic stiffness of multiphase nanocomposites.

\section{Conflict of Interests}

The authors declare that there is no conflict of interests regarding the publication of this paper.

\section{Acknowledgments}

The authors would like to thank Dr. Marie Bourgeaux-Goget for assistance with the experimental work. The authors would also like to thank Dr. Cato Dørum and Tomas Roll Frømyr for discussions and valuable input.

\section{References}

[1] S.-Y. Fu, X.-Q. Feng, B. Lauke, and Y.-W. Mai, "Effects of particle size, particle/matrix interface adhesion and particle loading 
on mechanical properties of particulate-polymer composites," Composites Part B: Engineering, vol. 39, no. 6, pp. 933-961, 2008.

[2] D. R. Paul and L. M. Robeson, "Polymer nanotechnology: nanocomposites," Polymer, vol. 49, no. 15, pp. 3187-3204, 2008.

[3] A. C. Taylor, "Adhesives with nanoparticles," in Handbook of Adhesion Technology, L. F. da Silva, A. Öchsner, and R. D. Adams, Eds., pp. 1437-1460, Springer, Berlin, Germany, 2011.

[4] B. H. Cipriano, A. K. Kota, A. L. Gershon et al., "Conductivity enhancement of carbon nanotube and nanofiber-based polymer nanocomposites by melt annealing," Polymer, vol. 49, no. 22, pp. 4846-4851, 2008.

[5] A. L. Gershon, D. P. Cole, A. K. Kota, and H. A. Bruck, "Nanomechanical characterization of dispersion and its effects in nano-enhanced polymers and polymer composites," Journal of Materials Science, vol. 45, no. 23, pp. 6353-6364, 2010.

[6] H. Zhang, Z. Zhang, K. Friedrich, and C. Eger, "Property improvements of in situ epoxy nanocomposites with reduced interparticle distance at high nanosilica content," Acta Materialia, vol. 54, no. 7, pp. 1833-1842, 2006.

[7] T. D. Fornes and D. R. Paul, "Modeling properties of nylon 6/clay nanocomposites using composite theories," Polymer, vol. 44, no. 17, pp. 4993-5013, 2003.

[8] J. N. Coleman, U. Khan, W. J. Blau, and Y. K. Gun'ko, "Small but strong: a review of the mechanical properties of carbon nanotube-polymer composites," Carbon, vol. 44, no. 9, pp. 1624$1652,2006$.

[9] T. H. Hsieh, A. J. Kinloch, K. Masania, A. C. Taylor, and S. Sprenger, "The mechanisms and mechanics of the toughening of epoxy polymers modified with silica nanoparticles," Polymer, vol. 51, no. 26, pp. 6284-6294, 2010.

[10] F. Fisher and L. C. Brinson, "Nanomechanics of nanoreinforced polymers," in Handbook of Theoretical and Computational Nanotechnology: Functional Nanomaterials, Nanoparticles, and Polymer Design, M. Rieth and W. Schommers, Eds., pp. 253-360, American Scientific Publishers, 2006.

[11] D. Srivastava, C. Wei, and K. Cho, "Nanomechanics of carbon nanotubes and composites," Applied Mechanics Reviews, vol. 56, no. 2, pp. 215-230, 2003.

[12] B. D. Agarwal, L. J. Broutman, and K. Chandrashekhara, Analysis and Performance of Fiber Composites, John Wiley \& Sons, 3rd edition, 2006.

[13] H. L. Cox, "The elasticity and strength of paper and other fibrous materials," British Journal of Applied Physics, vol. 3, no. 3, pp. 72-79, 1952.

[14] J. D. Fidelus, E. Wiesel, F. H. Gojny, K. Schulte, and H. D. Wagner, "Thermo-mechanical properties of randomly oriented carbon/epoxy nanocomposites," Composites Part A: Applied Science and Manufacturing, vol. 36, no. 11, pp. 1555-1561, 2005.

[15] T. Thorvaldsen, "A model study of the effective Young's modulus for randomly distributed short-fiber composites," FFI Report 2011/00212, 2011.

[16] L. J. Cohen and O. Ishai, "The elastic properties of three-phase composites," Journal of Composite Materials, vol. 1, no. 4, pp. 390-403, 1967.

[17] O. Ishai and L. J. Cohen, "Elastic properties of filled and porous epoxy composites," International Journal of Mechanical Sciences, vol. 9, no. 8, pp. 539-546, 1967.

[18] G. P. Tandon and G. J. Weng, "The effect of aspect ratio of inclusions on the elastic properties of unidirectionally aligned composites," Polymer Composites, vol. 5, no. 4, pp. 327-333, 1984.
[19] G. J. Weng, "Some elastic properties of reinforced solids, with special reference to isotropic ones containing spherical inclusions," International Journal of Engineering Science, vol. 22, no. 7, pp. 845-856, 1984.

[20] Y. P. Qiu and G. J. Weng, "On the application of Mori-Tanaka's theory involving transversely isotropic spheroidal inclusions," International Journal of Engineering Science, vol. 28, no. 11, pp. 1121-1137, 1990.

[21] B. Paul, "Prediction of elastic constants of multiphase materials," Transactions of the Metallurgical Society of AIME, vol. 218, pp. 36-41, 1960.

[22] G. P. Tandon and G. J. Weng, "Average stress in the matrix and effective moduli of randomly oriented composites," Composites Science and Technology, vol. 27, no. 2, pp. 111-132, 1986.

[23] J. C. Halpin and J. L. Kardos, "The Halpin-Tsai equations: a review," Polymer Engineering and Science, vol. 16, no. 5, pp. 344352, 1976.

[24] J. C. Halpin, "Stiffness and expansion estimates for oriented short fiber composites," Journal of Composite Materials, vol. 3, pp. 732-734, 1969.

[25] J. C. Halpin and N. J. Pagano, "The laminate approximation for randomly oriented fibrous composites," Journal of Composite Materials, vol. 3, no. 4, pp. 720-724, 1969.

[26] G. Swaminathan, M. Hossain, and K. N. Shivakumar, "Mechanical characterization of nanosilica/epoxy nanocomposites," in Proceedings of the 51st AIAA/ASME/ASCE/AHS/ASC Structures, Structural Dynamics, and Materials Conference, Orlando, Fla, USA, April 2010.

[27] T. Gómez-del Río, P. Poza, J. Rodríguez, M. C. García-Gutiérrez, J. J. Hernández, and T. A. Ezquerra, "Influence of singlewalled carbon nanotubes on the effective elastic constants of poly(ethylene terephthalate)," Composites Science and Technology, vol. 70, no. 2, pp. 284-290, 2010.

[28] T. Mura, Micromechanics of Defects in Solids, Martinus Nijhoff, The Hague, The Netherlands, 1982.

[29] T. Mori and K. Tanaka, "Average stress in matrix and average elastic energy of materials with misfitting inclusions," Acta Metallurgica, vol. 21, no. 5, pp. 571-574, 1973.

[30] J. D. Eshelby, "Elastic inclusions and inhomogeneities," in Progress in Solid Mechanics, I. N. Sneddon and R. Hill, Eds., vol. 2, pp. 89-140, North-Holland, Amsterdam, The Netherlands, 2 edition, 1961.

[31] J. D. Eshelby, "The determination of the elastic field of an ellipsoidal inclusion, and related problems," Proceedings of the Royal Society of London. Series A. Mathematical, Physical and Engineering Sciences, vol. 241, no. 1226, pp. 376-396, 1957.

[32] C. L. Tucker III and E. Liang, "Stiffness predictions for unidirectional short-fiber composites: review and evaluation," Composites Science and Technology, vol. 59, no. 5, pp. 655-671, 1999.

[33] C. Weinberger, W. Cai, and D. Barnett, Lecture Notes-Elasticity of Microscopic Structures, ME340B, Stanford University, 2004.

[34] T. Thorvaldsen, "Modeling the elastic stiffness of nanocomposites using the Mori-Tanaka method," FFI Report 2014/00494, 2014.

[35] F. T. Fisher, R. D. Bradshaw, and L. C. Brinson, "Fiber waviness in nanotube-reinforced polymer composites-I: modulus predictions using effective nanotube properties," Composites Science and Technology, vol. 63, no. 11, pp. 1689-1703, 2003.

[36] T. B. Lewis and L. E. Nielsen, "Dynamic mechanical properties of particulate-filled composites," Journal of Applied Polymer Science, vol. 14, no. 6, pp. 1449-1471, 1970. 
[37] S. McGee and R. L. McGullough, "Combining rules for predicting the thermoelastic properties of particulate filled polymers, polymers, polyblends, and foams," Polymer Composites, vol. 2, no. 4, pp. 149-161, 1981.

[38] A. P. Holt, P. J. Griffin, V. Bocharova et al., "Dynamics at the polymer/nanoparticle interface in poly(2-vinylpyridine)/silica nanocomposites," Macromolecules, vol. 47, no. 5, pp. 1837-1843, 2014.

[39] G. M. Odegard, T. C. Clancy, and T. S. Gates, "Modeling of the mechanical properties of nanoparticle/polymer composites," Polymer, vol. 46, no. 2, pp. 553-562, 2005.

[40] M. L. Dunn and H. Ledbetter, "Elastic moduli of composites reinforced by multiphase particles," Journal of Applied Mechanics, vol. 62, no. 4, pp. 1023-1028, 1995.

[41] D.-L. Shi, X.-Q. Feng, Y. Y. Huang, K.-C. Hwang, and H. Gao, "The effect of nanotube waviness and agglomeration on the elastic property of carbon nanotube-reinforced composites," Journal of Engineering Materials and Technology, vol. 126, no. 3, pp. 250-257, 2004.

[42] D.-L. Shi, X.-Q. Feng, Y. Y. Huang, and K.-C. Hwang, "Critical evaluation of the stiffening effect of carbon nanotubes in composites," Key Engineering Materials, vol. 261-263, pp. 1487$1492,2004$.

[43] R. M. Jones, Mechanics of Composite Materials, Taylor \& Francis, Philadelphia, Pa, USA, 1999.

[44] B. B. Johnsen, M. Bourgeaux-Goget, and T. Olsen, "Material properties of silica/epoxy nanocomposites," FFI Report 2015/00366, 2015.

[45] S. Sprenger, "Personal communication," 2014.

[46] ASTM, "Standard test methods for density and specific gravity (relative density) for plastics by displacement," ASTM D792-08, 2008.

[47] International Organization for Standardization, "Plasticsdetermination of tensile properties-part 1: general principles," ISO 527-1:2012, International Organization for Standardization, London, UK, 2012.

[48] International Organization for Standardization, "Plasticsdetermination of tensile properties-part 2: test conditions for moulding and extrusion plastics," International Organization for Standardization 527-2:1996, 1996.

[49] F. Meyer, G. Sanz, A. Eceiza, I. Mondragon, and J. Mijović, "The effect of stoichiometry and thermal history during cure on structure and properties of epoxy networks," Polymer, vol. 36, no. 7, pp. 1407-1414, 1995.

[50] Y.-L. Liu, C.-Y. Hsu, W.-L. Wei, and R.-J. Jeng, "Preparation and thermal properties of epoxy-silica nanocomposites from nanoscale colloidal silica," Polymer, vol. 44, no. 18, pp. 51595167, 2003.

[51] S. Torquato and F. Lado, "Effective properties of two-phase disordered composite media: II. Evaluation of bounds on the conductivity and bulk modulus of dispersions of impenetrable spheres," Physical Review B, vol. 33, no. 9, pp. 6428-6435, 1986.

[52] S. Torquato, "Nearest-neighbor statistics for packings of hard spheres and disks," Physical Review E, vol. 51, no. 4, pp. 31703182, 1995.

[53] J. Karger-Kocsis and Z. Zhang, "Structure-property relationships in nanoparticle/semicrystalline thermoplastic composites," in Mechanical Properties of Polymers based on Nanostructure and Morphology, G. H. Michler and F. J. Baltá-Calleja, Eds., pp. 553-602, Taylor \& Francis Group, 2005.
[54] B. B. Johnsen, A. J. Kinloch, R. D. Mohammed, A. C. Taylor, and S. Sprenger, "Toughening mechanisms of nanoparticlemodified epoxy polymers," Polymer, vol. 48, no. 2, pp. 530-541, 2007.

[55] T. R. Frømyr, M. Bourgeaux-Goget, and F. K. Hansen, "A comparative study of the dispersion of multi-wall carbon nanotubes made by arc-discharge and chemical vapour deposition," Journal of Nanoscience and Nanotechnology, vol. 15, no. 5, pp. 3496-3506, 2015.

[56] C. Antonelli, B. Serrano, J. Baselga, R. Ozisik, and J. C. Cabanelas, "Interfacial characterization of epoxy/silica nanocomposites measured by fluorescence," European Polymer Journal, vol. 62, pp. 31-42, 2015.

[57] R. Andrews, D. Jacques, M. Minot, and T. Rantell, "Fabrication of carbon multiwall nanotube/polymer composites by shear mixing," Macromolecular Materials and Engineering, vol. 287, no. 6, pp. 395-403, 2002.

[58] J. P. Lu, "Elastic properties of carbon nanotubes and nanoropes," Physical Review Letters, vol. 79, no. 7, pp. 1297-1300, 1997. 

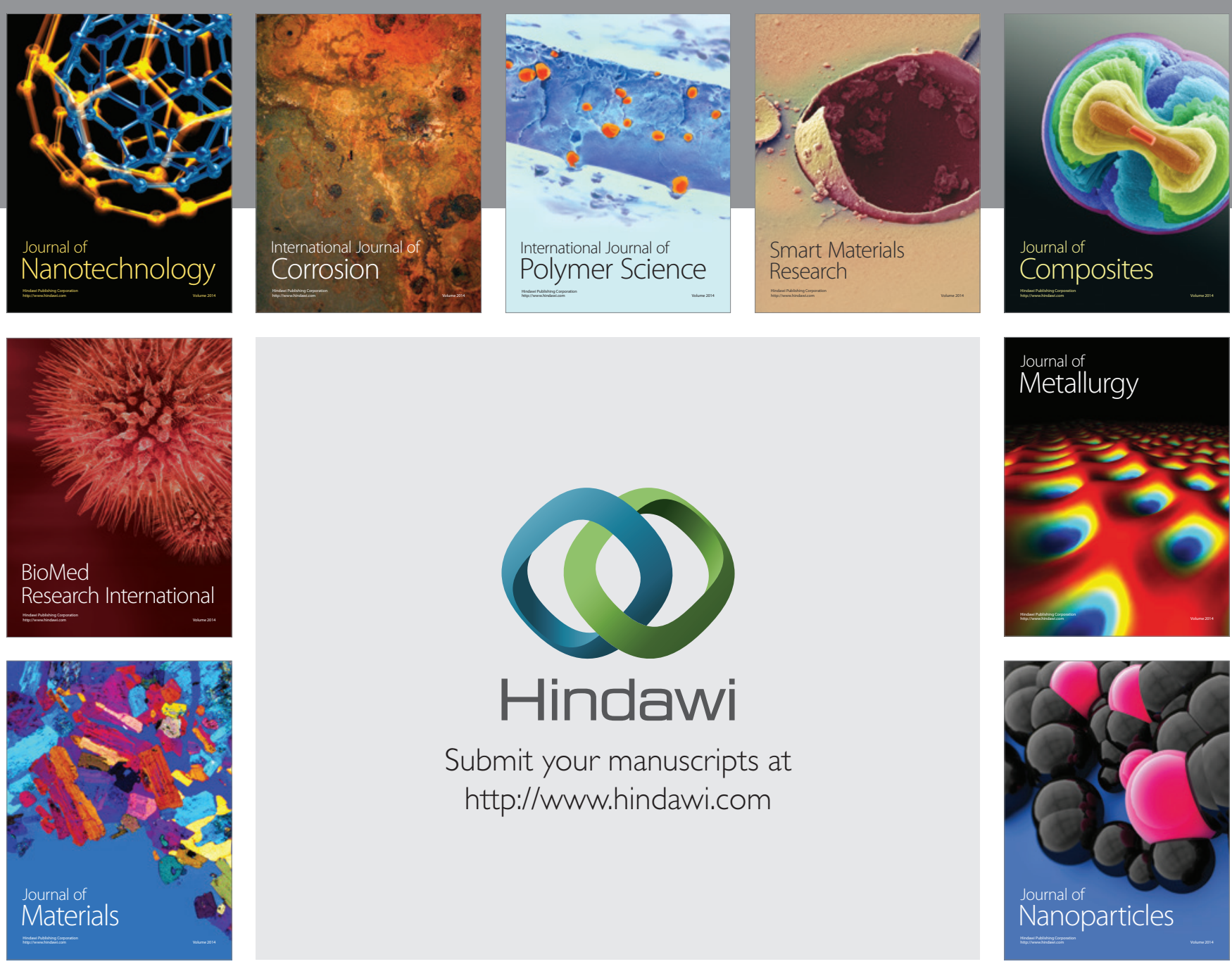

Submit your manuscripts at http://www.hindawi.com
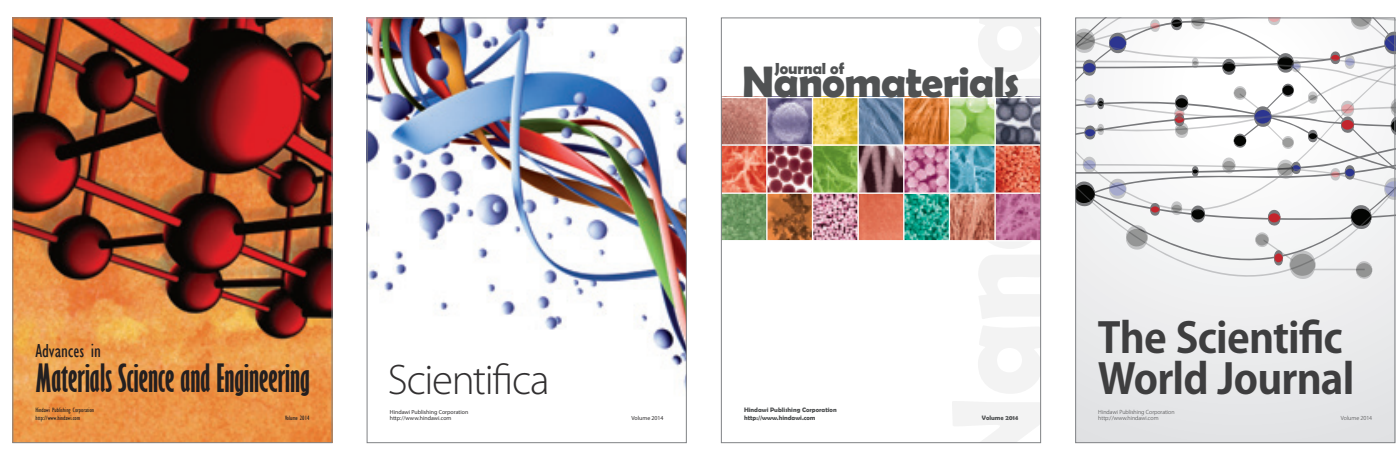

\section{The Scientific World Journal}
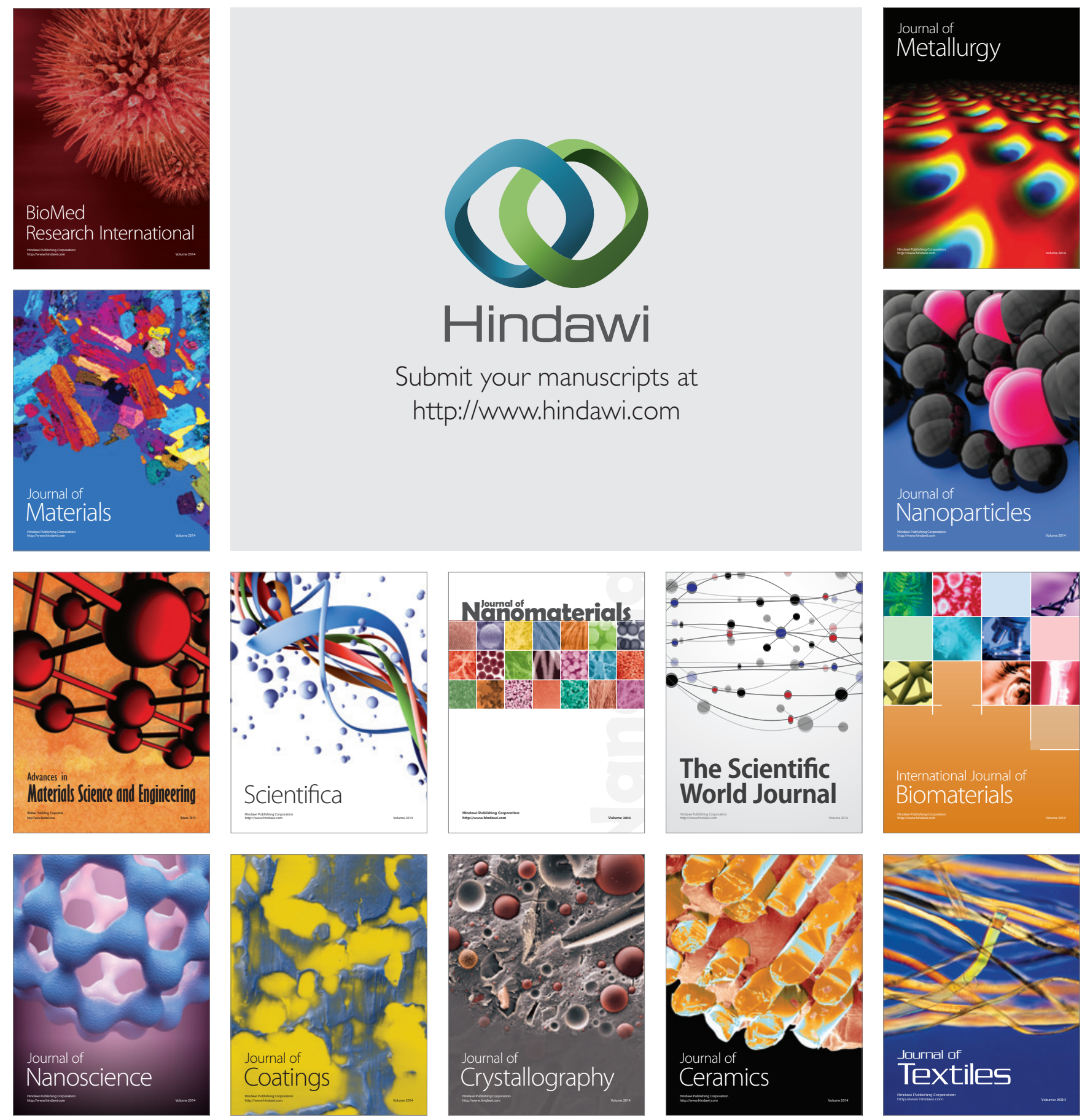